Nouvelles perspectives en sciences sociales

Revue internationale de systémique complexe et d'études relationnelles

\title{
Regard d'artistes sur le territoire : Balle perdue, une visite-performance de Dector et Dupuy
}

\section{Claire Le Thomas}

Volume 10, numéro 1, novembre 2014

Sur le thème du territoire

URI : https://id.erudit.org/iderudit/1028441ar

DOI : https://doi.org/10.7202/1028441ar

Aller au sommaire du numéro

Éditeur(s)

Prise de parole

ISSN

1712-8307 (imprimé)

1918-7475 (numérique)

Découvrir la revue

Citer cet article

Le Thomas, C. (2014). Regard d'artistes sur le territoire : Balle perdue, une visite-performance de Dector et Dupuy. Nouvelles perspectives en sciences sociales, 10(1), 237-281. https://doi.org/10.7202/1028441ar
Résumé de l'article

Balle perdue est une performance réalisée par deux artistes, Dector et Dupuy, en 2014. Il s'agit d'une visite guidée des quinze kilomètres compris entre Caen et la Manche. Au moyen du concept d'objet d'art-lieu théorisé par Anne Volvey, cet article analyse les effets de cette oeuvre sur la perception spatiale des spectateurs et la manière dont elle fabrique du territoire, interrogeant en retour la complexité de cette notion en géographie. 


\section{Regard d'artistes sur le territoire : Balle perdue, une visite-performance de Dector et Dupuy}

Claire Le Thomas

LAHIC, CNRS, Paris

\section{Des artistes, une exposition et un espace}

D ector et Dupuy sont deux artistes dont l'œuvre s'appuie sur leur environnement: ils réalisent des séries photographiques attirant l'attention des spectateurs sur des gestes urbains insolites (caviardages, dépôts, coincements, empêchements, slogans $\left.{ }^{1} . ..\right)$; ils contrarient l'aspect immaculé du musée ou de la galerie dans leurs installations; leurs performances reposent sur la description d'éléments autour d'eux ${ }^{2}$. Ils ont en particulier développé une forme artistique au propos géographique: des visites guidées de lieux, de quartiers ou de villes (par exemple le

$\overline{1 \quad \text { Les caviardages sont les couches de peinture recouvrant les graffitis ou les }}$ traits qui les biffent pour les rendre illisibles. Les photos de dépôts montrent des ordures déposées de manière originale (dans le coin d'une fenêtre, sur une sculpture, sur un piédestal...), les coincements celles coincées intentionnellement (entre les barreaux d'une grille, les branches d'un arbre, dans un grillage...). Les empêchements inventorient des dispositifs matériels (publics ou privés) destinés à empêcher de circuler, de s'asseoir... Les slogans rassemblent des inscriptions murales à caractère politique.

2 Pour plus d'informations sur le travail de ces artistes, voir leur site internet: http://www.dector-dupuy.com/; site consulté en septembre 2014. 
jardin d'agronomie tropicale, le quartier du marais à Paris, Versailles, Noisiel...) ayant pour objectif de renouveler la perception spatiale des spectateurs. Balle perdue est l'une de ces visites-performances ${ }^{3}$ réalisée au cours de l'atelier "Art contemporain et pratiques géographiques: territoires en représentation». Cette journée d'étude se proposait d'analyser l'espace d'implantation d'une exposition d'art contemporain organisée par l'école supérieure d'arts et médias (ésam) Caen/ Cherbourg, le musée éclaté de la presqu'île de Caen (MéPIC). Le principe de cette exposition, très liée aux préoccupations économiques et politiques locales ${ }^{4}$, était d'inviter quinze artistes à réaliser quinze ouvres éphémères présentées dans quinze communes de la basse vallée de l'Orne (voir la figure 1). Le MéPIC s'étendait donc sur l'espace constitué par les quinze communes partenaires, une zone estuarienne de $15 \mathrm{~km}$ de long comprise entre Caen et la Manche, dénommée "presqu'île de Caen " pour l'occasion. Cet espace, marqué par la présence forte de l'eau (canal, Orne, marais, plages...) et l'influence caennaise, est un territoire de projet que les acteurs politiques et économiques tentent de transformer en territoire - et c'est d'ailleurs précisément l'un des objectifs du musée éclaté que d'en favoriser l'émergence $^{6}$ - en stimulant une prise de conscience chez les habitants

Elle s'est déroulée le 19 juin 2013 de 9 h30 à 13 h30.

4 Pour en savoir plus sur le MéPIC et son inscription territoriale, voir le site de l'exposition (http://www.mepic.fr/; site consulté en septembre 2014) et le texte de Claire Le Thomas, ( Art de l'entre-deux: le MéPIC au service de la reconversion portuaire de Caen", Urbanités, "Repenser la ville portuaire», $\mathrm{n}^{\circ}$ 4, novembre 2014, [en ligne] http://www.revue-urbanites.fr/, site consulté en septembre 2014, à paraître).

5 Amfréville, Bénouville, Biéville-Beuville, Blainville-sur-Orne, Caen, Colleville-Montgoméry, Colombelles, Hermanville-sur-Mer, HérouvilleSaint-Clair, Merville-Franceville, Mondeville, Ouistreham, Ranville, Saint Aubin d'Arquenay, Sallenelles.

6 Dans l'esprit de la manifestation, il s'agit en effet de donner une réalité et une identité à ce territoire encore inexistant. Projet culturel porté par une volonté politique de dynamisation régionale, le MéPIC reflète ainsi la fabrique territoriale mise en ouvre par les élus et les acteurs économiques pour exploiter le potentiel de cet espace portuaire en recherche de nouvelles vocations. Elle procède à la manière d'Estuaire à Nantes (cf. «Estuaire 2009. Le paysage, l'art et le fleuve», 303 arts recherches créations, La revue culturelle 
et les entreprises des liens qui unissent ces quinze villes et de leur avenir commun. Après avoir été principalement occupée par des complexes industrialo-portuaires, cette zone estuarienne est progressivement devenue, sous l'effet de la désindustrialisation et de l'urbanisation, banlieue résidentielle périurbaine de Caen, conduisant à l'exploitation croissante du potentiel récréatif et balnéaire de l'eau. Le nom "presqu'île de Caen" donné par l'exposition est l'extension d'une appellation existante: pour les caennais, la Presqu'île correspond à l'espace délimité par l'Orne, le canal de Caen à la Mer et le viaduc de Calix (voir la figure 2), l'élargissement lexical proposé par le musée éclaté prolongeant un phénomène à l'œuvre depuis quelques années avec les projets de réaménagement des friches de la Presqu'île au sens strict et des zones limitrophes ${ }^{7}$.

des Pays de la Loire, n 106, 2009 et «Estuaire 2012. Le paysage, l'art et le fleuve", 303 arts recherches créations, La revue culturelle des Pays de la Loire, $\mathrm{n}^{\circ}$ 122, 2012).

7 Voir à ce sujet le site de la Société publique locale d'Aménagement (SPLA) Caen-Presquîle (http://www.caen-presquile.com/; site consulté en septembre 2014) et «La presqu'île de Caen", Ohm, un petit journal de l'art contemporain (école régionale des Beaux-arts Caen la mer), n 23, mars 2005. 
$240 \quad$ NPSS, VOLUME 10, NUMÉRO 1, 2014

Figure 1

Carte du MéPIC

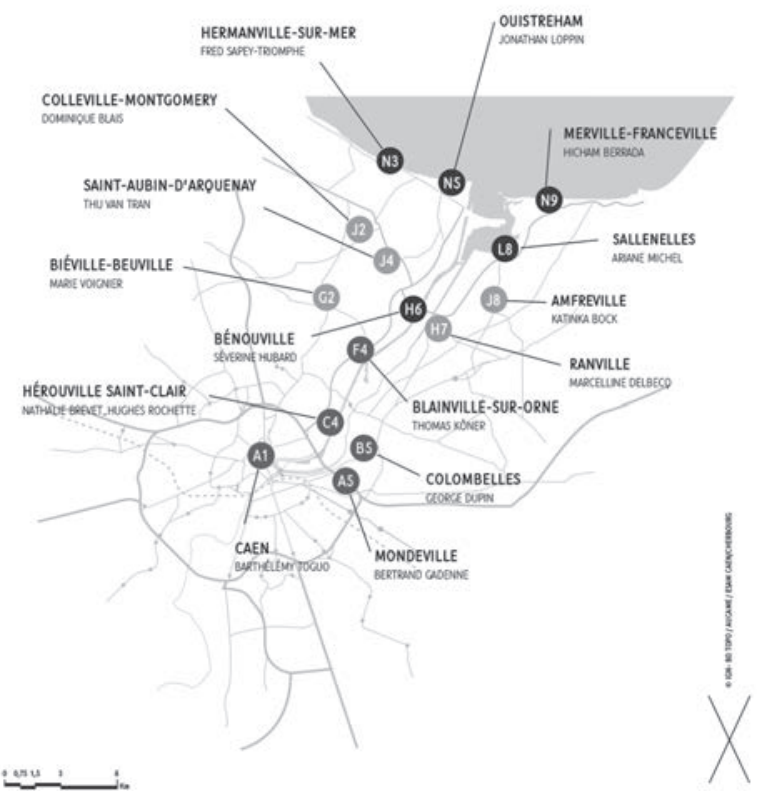

Une exposition implantée sur quinze communes bordant l'estuaire de l'Orne. CIGN / Aucame / ésam Caen/Cherbourg

Figure 2

La presqu'île de Caen

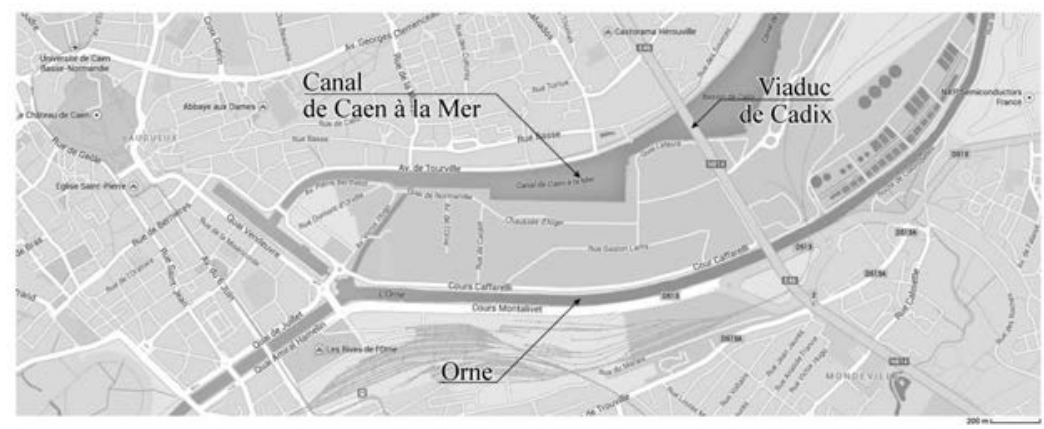

Au sens strict, la Presqu'île est délimitée par l'Orne, le canal de Caen à la Mer et le viaduc de Calix.

(c) Données cartographiques Google 2014

(c) Claire Le Thomas 
En demandant à des artistes de se confronter à l'espace sur lequel se déploie le MéPIC, il s'agissait d'interroger la propension de l'art à produire des connaissances géographiques - au sens de réflexion sur les relations qui s'établissent entre les hommes et l'espace - et de faire dialoguer la vision et l'expérience artistiques avec celles de la recherche. Le choix s'est porté sur Dector et Dupuy, car le propre de leurs visites est de livrer des interprétations spatiales et d'utiliser pour ce faire un regard informé dans la mesure où il convoque l'art, l'histoire et tous les savoirs récoltés pendant les repérages et l'enquête préliminaire. En conséquence, si leurs visites expriment leur perception des espaces traversés pendant la performance, elles croisent dans le même temps des représentations variées puisqu' elles s'appuient sur les informations fournies par différents acteurs (habitants, élus, passants, organisateurs du MéPIC, scientifiques...). Au cours de ces circuits, les artistes décrivent et commentent des éléments autour d'eux; ils cherchent de la sorte à engendrer dans l'auditoire une expérience esthétique qui met en évidence les composantes environnementales, imaginaires, mémorielles ou sociales de l'entourage des spectateurs. En d'autres termes, Dector et Dupuy travaillent avec les lieux et font travailler les lieux lors de leurs visites guidées. Ils travaillent avec les lieux car leur matériau de création est constitué de leur environnement immédiat, ce qui explique l'importance des repérages dans leur pratique artistique. Ces temps de découverte et de reconnaissance sont le préalable, le point de départ de la création; la narration élaborée par les artistes naît et s'appuie sur les caractéristiques de l'espace parcouru, elle est un "énoncé de terrain $^{8} »$. Ils font travailler les lieux car ils engagent par leurs commentaires un processus de révélation et de transformation: ils révèlent les strates sensibles ou historiques qui composent un lieu et déterminent son aménagement ou ses usages; ce faisant, ils transforment la perception spatiale des auditeurs. Dector et Dupuy médiatisent les relations entre un public et son environnement

8 Cf. Myriam Houssay-Holzschuch et Anne Volvey, «La rue comme palette. La Pietà sud-africaine, Soweto-Warwick, mai 2002, Ernest Pignon-Ernest ", Travaux de l'Institut de Géographie de Reims, n 129-130, 2008, p. 137-161. 
pour créer un objet d'art-lieu', c'est-à-dire une œuvre triple qui englobe tout à la fois un lieu, la glose des artistes et ses effets sur l'entendement des spectateurs, à savoir la nouvelle représentation qui naît de la conjonction des deux premiers. Les visites de Dector et Dupuy possèdent ainsi une valeur performative ${ }^{10}$ qui repose sur la parole. L'énoncé des artistes n'est pas uniquement descriptif, il agit sur les spectateurs, mais la modification à l'œuvre est d'ordre sémantique, dans la mesure où elle ne modifie pas l'espace en lui-même mais la grille de lecture avec laquelle les visiteurs l'appréhendent. De la sorte, parce qu'elle passe par le verbe, par une sémiotisation de l'espace, qu'elle repose sur une appropriation artistique, celle de Dector et Dupuy, et qu'elle relève d'une prise de conscience, cette modification peut s'apparenter à un processus de territorialisation pour les spectateurs: elle crée « un ensemble approprié, conscientisé, réordonné par ce qui relève de la sémiophère ${ }^{11}$ ». Des artistes fabriquant du territoire intervenant sur un espace de projet en voie de territorialisation: Balle perdue semble à même de questionner ce concept primordial en géographie ${ }^{12}$.

Quelle exégèse ont donc élaboré ces artistes sur l'espace du musée éclaté et par quels moyens plastiques l'ont-ils exprimée? Ils ont conçu une visite alternant commentaires depuis un bus et parcours à pied autour de six zones bien différenciées - la presqu'île de Caen proprement dite, le plateau de Colombelles, le plain d'Amfréville, les dunes et marais salants de Merville-

$\overline{9} \quad$ Pour reprendre le concept d'Anne Volvey. Cf. Anne Volvey, «LAND ARTS. Les fabriques spatiales de l'art contemporain", Travaux de l'Institut de Géographie de Reims, n 129-130, 2008, p. 3-25.

10 John Langshaw Austin, Quand dire c'est faire, Paris, Seuil, 1970 [éd. anglaise, 1962].

11 Bernard Elissalde, «Une géographie des territoires», L'information géographique, vol. 66, n 3, 2002, p. 198.

12 Pour cette réflexion sur le territoire, je me base sur les titres suivants : Roger Brunet, Robert Ferras et Hervé Théry (dir.), Les mots de la géographie, dictionnaire critique, $2^{\mathrm{e}}$ ed. revue, Montpellier/Paris, GIP RECLUS/La documentation française, 1992; Bernard Elissalde, loc. cit. et Régis Keerle et Raymonde Séchet, "Petite histoire de nos délicatesses avec le territoire», ESO, Travaux et documents de l'Unité Mixte de Recherche 6590, Espaces et sociétés, n² 26, septembre 2007, p. 7-15. 
Franceville, les résidences pavillonnaires de Bénouville et la Pointe du Siège à Ouistreham (voir la figure 3) - qui résument les particularités de la basse vallée de l'Orne et de l'agglomération caennaise. Les friches industrialo-portuaires de la Presqu'île et du plateau de Colombelles témoignent de sa richesse et de son éclat passés; les deux sites de dunes et de marais protégés sont typiques du littoral et des espaces naturels estuariens; parmi ceux-ci, la Pointe du Siège évoque la composante balnéaire de certaines communes du MéPIC et les nouveaux usages de l'eau qui s'ébauchent; les deux zones d'habitation illustrent par leur urbanisme antithétique le passage d'une société rurale à une société urbaine et l'évolution des modes de vie et d'urbanisme qui l'accompagne ${ }^{13}$. Chaque partie du circuit a été l'occasion de déciller le regard des spectateurs, d'amplifier leurs sensations pour faire éclore une émotion esthétique liée à la nature des espaces parcourus ou aux dimensions idéelles et mémorielles les traversant. De la sorte, Balle perdue, tout en proposant une expérience du territoire ${ }^{14}$ du MéPIC tel que pratiqué, vécu, ressenti et représenté par Dector et Dupuy - expérience qui révèle certains de ses enjeux socio-économiques - examine les rapports que nous entretenons avec notre environnement. C'est donc la manière dont Balle perdue est productrice de savoirs sur cet espace et interroge en retour sa territorialité que cet article met en lumière à travers l'analyse de cette performance.

\footnotetext{
$\overline{13}$ Il ne manque à cet inventaire que la permanence de l'affectation portuaire de cet espace sur laquelle l'article reviendra.

14 Par commodité, j'utiliserai le terme de territoire pour parler de l'espace délimité par les quinze communes partenaires du MéPIC. Il est en effet envisagé comme tel par la manifestation et par les acteurs politiques à l'origine du projet, même si la réalité de ce territoire pour les habitants et l'effectivité de cette triple fabrique territoriale - celle du projet d'aménagement, du musée éclaté, et enfin de Dector et Dupuy - reste à démontrer.
} 
Figure 3

Carte de Balle perdue

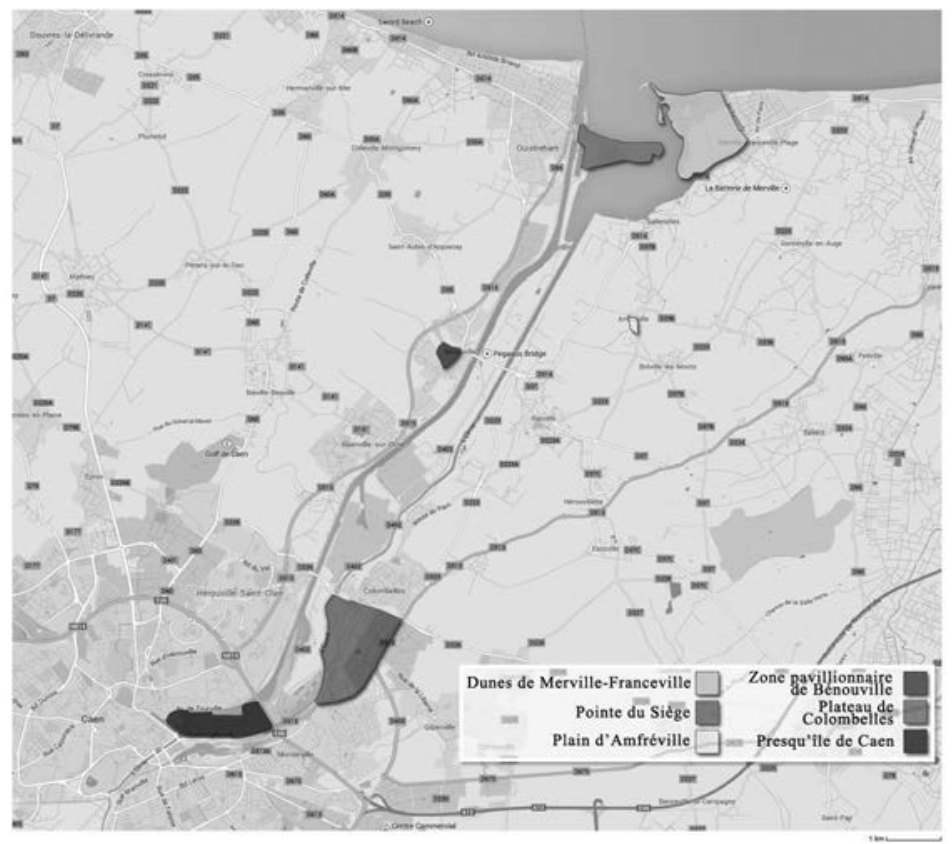

Les six zones sur lesquelles Dector et Dupuy sont intervenus pour leur performance Balle perdue.

() Données cartographiques Google 2014

(c) Claire Le Thomas

Recrutée en tant que coordinatrice scientifique du musée éclaté, mon rôle consistait à développer un programme de recherche autour de l'exposition. J'ai donc été commanditaire de cette œuvre, mais également informatrice privilégiée des artistes. Pour mener à bien ma mission, j'ai en effet réalisé un terrain ${ }^{15}$ afin d'identifier les problématiques à étudier et les chercheurs et

$\overline{15}$ En référence à la pratique ethnographique : j’ai rencontré des acteurs, je me suis documentée, $j$ 'ai parcouru l'espace de l'exposition et j'y ai vécu. Ce terme de "terrain" met également l'accent sur la place d'observatrice participante qui a été la mienne au sein du musée éclaté et du processus de production de Balle perdue. Il entre enfin en résonance avec mon rôle d'informatrice privilégiée pour les artistes (la particularité de l'ethnologie étant de se reposer en grande partie sur des informateurs pour son analyse des sociétés) - autre renversement de position que ma mission impliquait. 
artistes à solliciter. J'ai partagé les connaissances forgées en ces circonstances avec Dector et Dupuy en leur servant de guide pendant leurs repérages et en organisant des rencontres, auxquelles j'ai assisté, avec des acteurs de cet espace ${ }^{16}$. J'ai ainsi pu distinguer, parmi les matériaux accumulés lors du travail préparatoire, ce qu'ils avaient sélectionné et leur façon de les utiliser lors de la visite guidée. Mon expérience de Balle perdue et nos conversations sur leur démarche et leurs méthodes de travail nourrissent également ma réflexion. Pour cet article, la chercheuse que je suis et que j'étais dans le cadre du projet, avec sa connaissance objectivée, tant de la zone d'implantation du musée éclaté que de l'œuvre de Dector et Dupuy, revient donc sur son vécu de spectatrice ${ }^{17}$ en utilisant la compréhension accrue que lui a offert sa position de protagoniste du processus de création et son contact avec les artistes.

\section{Poétiser le réel, un processus d'artification}

Le premier artifice employé par Dector et Dupuy pour agir sur le regard des spectateurs consiste à poétiser le réel. Ils engagent de la sorte un processus d'artification ${ }^{18}$, c'est-à-dire une transformation de la réalité en art, qui constitue un premier niveau d'action sur l'espace du MéPIC: il devient une œuvre d'art, un objet valorisé à fort prestige. Si cet acte n'est pas porteur en soi de savoirs géographiques sur cet environnement, il est le préalable aux autres opérations des artistes et signale en retour l'une de ses particularités - le manque d'attractivité de certains lieux en friche

$\overline{16}$ Karine Le Petit, Chargée de mission ethnologie au Centre régional de Culture ethnologique et technique (CRéCET) et responsable d'une enquête ethnographique sur les métiers portuaires à Caen et Cherbourg (http://www. metiers-portuaires.fr/; site consulté en septembre 2014 ); Marc Pottier, maire-adjoint en charge de la culture à Colombelles et directeur de l'Agence régionale de Conservation de l'Image et du Son de Basse-Normandie (ARCIS); Raymond Garnier, président de l'Association de Défense de l'Environnement et du Patrimoine de Merville-Franceville (ADEPMF).

17 N'ayant pas enquêté en ce sens, je ne suis en revanche pas en mesure d'évaluer les effets concrets de la performance sur les représentations spatiales des autres visiteurs.

18 Le terme est emprunté à Nathalie Heinich et Roberta Shapiro (dir.), De l'artification. Enquêtes sur le passage à l'art, Paris, EHESS, 2012. 
comme le Plateau ou la Presqu'île au sens strict, voire le discrédit qui les frappe.

À plusieurs reprises Dector et Dupuy se sont donc arrêtés sur des éléments sans qualité, qui passeraient inaperçus aux yeux des autres: un panneau de signalisation, un trou dans une haie, une zone d'activité. Passé au crible du regard des artistes, le banal sort de son anonymat et s'extrait de son statut de chose sans intérêt: ainsi, les noms des entreprises du Plateau, lus à voix haute au rythme de leur défilement dans le car, se convertissent en litanie poétique; un panneau d'interdiction à demi effacé devient une "nuit étoilée ${ }^{19}$ " qui s'accorde avec le parterre de fleurs à ses pieds (voir la figure 4). Leur sensibilité artistique les porte à interroger et disséquer leur environnement, à en percevoir les incongruités; il suffit alors à Dector et Dupuy de montrer et de décrire l'objet de leur étonnement pour que celui-ci devienne captivant. C'est par exemple une énigme qui a attiré l'attention des artistes sur le panneau de signalisation décrit ci-dessus: pourquoi est-il entouré d'un fil barbelé? Est-ce une protection contre le vol? Contre le vandalisme? Mais qui voudrait détruire un panneau déjà abîmé par le temps? De fil en aiguille, le mystère relevé par Dector et Dupuy se résout sous les yeux des spectateurs qui revivent les étapes du raisonnement des artistes: le fil de fer entoure en réalité le poteau qui supporte le panneau; il s'agit d'un poteau électrique dont certains fils pendent; le fil de fer sert à empêcher de grimper au poteau pour se brancher illégalement sur le réseau d'électricité... Restituées sous la forme d'une narration, cette monstration et cette analyse du réel sont un prétexte à raconter une histoire qui enchante la réalité et transporte les spectateurs dans une autre dimension. Au terme de ce récit, le panneau a vu ses qualités plastiques reconnues: une inversion de couleurs où ce qui devrait être rouge est blanc et ce qui devrait être bleu est rouge et rempli

19 Toutes les citations sans références sont extraites des notes de travail des artistes: ceux-ci rédigent en effet en totalité les commentaires de leur visite et gardent ces notes en main pendant la performance. Je les remercie d'avoir partagé avec moi ce document. Michel Dector et Michel Dupuy, Visite guidée du 19 juin 2013 Caen Ouistreham, Balle perdue, texte dactylographié, 2013. 
de points blanc; il est transformé en objet esthétique qui peut être contemplé pour sa beauté à l'instar d'une nuit étoilée.

Figure 4

La nuit de l'interdit

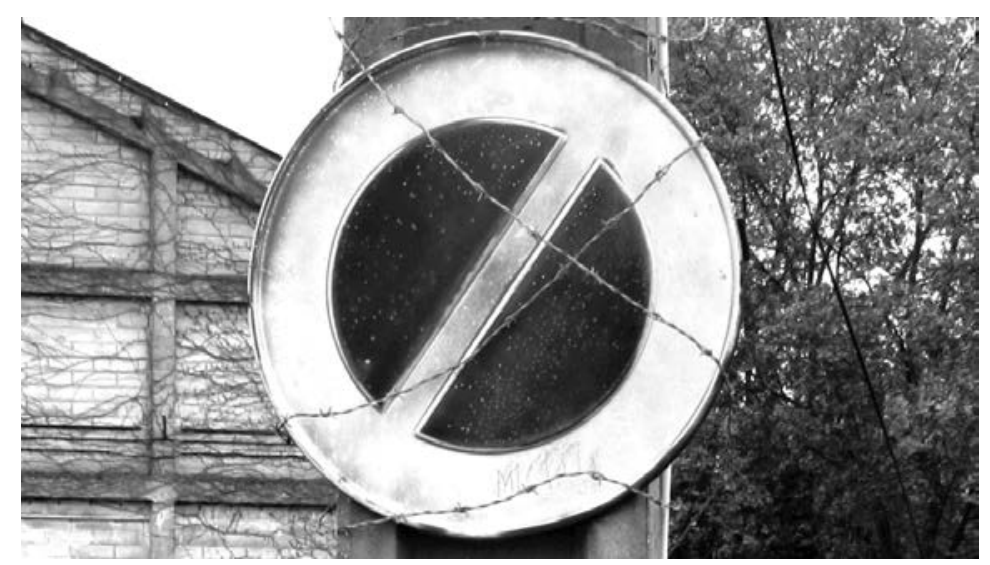

Sur la presqu'île de Caen, un panneau d'interdiction à demi effacé devient une "nuit étoilée".

\section{(C) Dector et Dupuy}

Bien souvent, ce sont des connaissances, des savoirs acquis au cours de l'enquête et des recherches qui précèdent leurs interventions artistiques, qui permettent d'opérer ce renouvellement du regard. Dector et Dupuy utilisent une des caractéristiques de toute visite guidée, à savoir la transmission de connaissances et la perception aiguisée qui en découle, pour l'adapter à des objets incongrus ou invisibles et engager de la sorte une valorisation du réel. La description savante d'un poteau rongé jusqu'au fer (voir la figure 5) amène par exemple les artistes à conter l'histoire du béton armé:

Remarquez le joli poteau sur la droite comme un totem. Il est usé par le temps et le sel, le fer enserre le béton fait de ciment et de granulats bruns. C'est comme un éclaté qui révèle l'intérieur de cette matière et la technique du béton armé. Le béton armé a été inventé par un jardinier autodidacte, un français nommé Joseph Monier qui cherchait à faire des pots à orangers moins chers et plus résistants. C'est plus tard François Hennebique qui déposera un brevet en 1892. 
Figure 5

Totem en béton

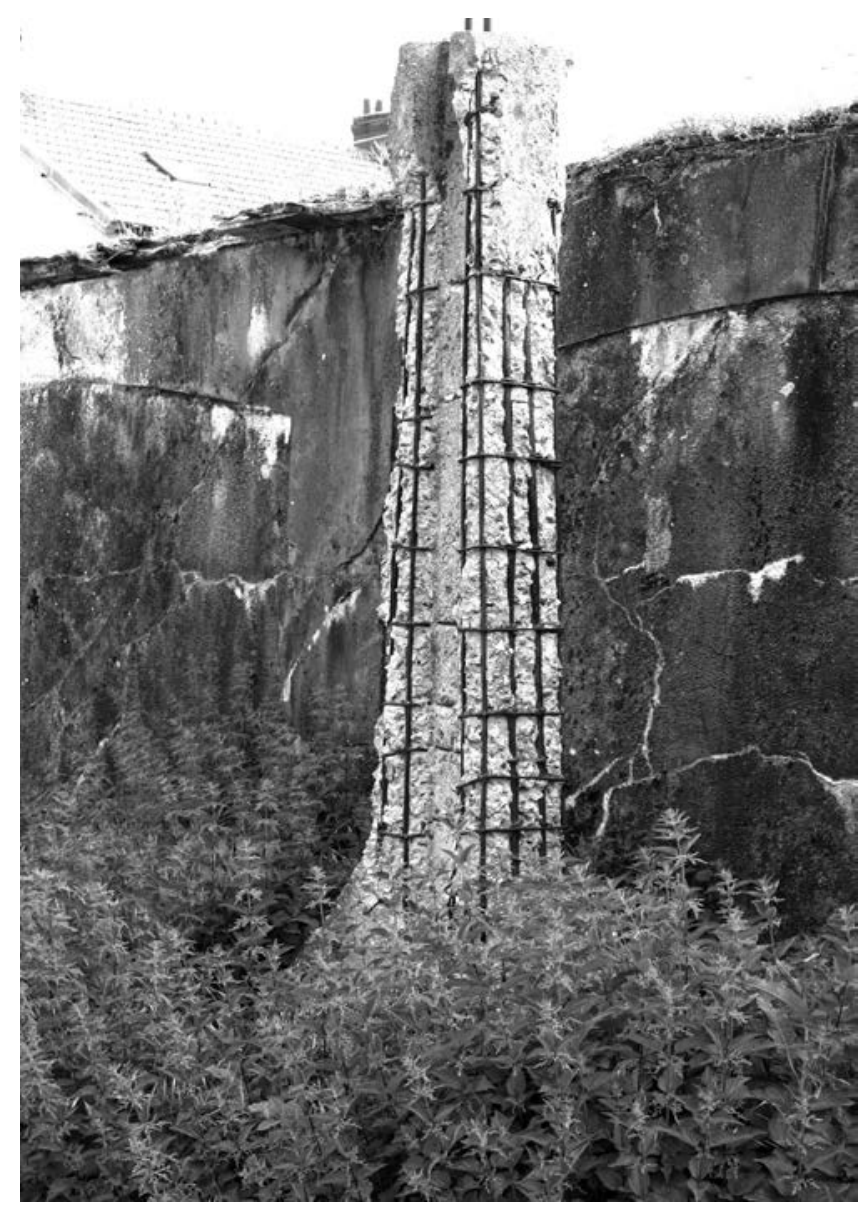

Poteau rongé jusqu'au fer sur la presqu'île de Caen. "Remarquez le joli poteau sur la droite comme un totem. II est usé par le temps et le sel, le fer enserre le béton fait de ciment et de granulats bruns. C'est comme un éclaté qui révèle l'intérieur de cette matière et la technique du béton armé. "

(c) Dector et Dupuy

Par leurs commentaires, Dector et Dupuy convertissent un simple poteau - et même un aménagement urbain tellement détérioré qu'il ne peut plus remplir la fonction pour laquelle il a été fabriqué - en témoignage de l'invention d'un matériau révolutionnaire pour l'architecture. Le récit, en s'achevant sur 
une citation de Tadao Ando, qui voyait dans le béton armé le marbre du $\mathrm{XX}^{\mathrm{e}}$ siècle, provoque un processus d'esthétisation similaire à celui à l'œuvre dans La nuit de l'interdit (voir la figure 4). Le poteau devient un objet précieux, voire une œuvre d'art digne d'admiration puisque le marbre, matériau noble et rare, a essentiellement servi à fabriquer sculptures, décors architecturaux et autres créations plastiques.

Le recours à des comparaisons artistiques pour poétiser le réel confirme ce qui se joue implicitement dans cette transfiguration du banal. En modifiant le regard des spectateurs sur des objets $a$ priori sans qualité, il ne s'agit pas seulement de rétablir une curiosité pour notre environnement quotidien, d'aiguiser notre perception, mais également de révéler l'artistique dans la réalité ou, pour le dire autrement, de dévoiler l'universalité du geste esthétique, un dessein déjà visible dans les séries des caviardages, des dépôts ou des coincements (voir les figures 6a, 6b et 6c). Que ce geste soit involontaire, le fait du hasard, il n'en est pas moins lu par Dector et Dupuy comme la trace d'une intentionnalité artistique, au sens où l'art surgit sans prévenir là où on ne l'attend pas, à l'image de cette composition abstraite formée par le dessin de parkings derrière l'école supérieure des arts et médias de Caen (voir la figure 7). La bordure de ciment séparant la pelouse du goudron forme des retraits réguliers, un motif d'escalier accentué par la séparation colorée. Devant ce tracé linéaire, des cubes de granit ont été disposés en arc de cercle. Cet agencement, outre son caractère pictural et sculptural, indique un changement d'usage - des emplacements de parking ont été remplacés par une courbe qui empêche de stationner et délimite une zone de circulation. Cette transformation du dessein/dessin à proximité de l'école d'art est alors interprétée par les artistes comme un repentir:

Un repentir, c'est un terme utilisé dans les arts plastiques pour indiquer une reprise, une amélioration du dessin, de la composition. Le repentir sur une feuille d'étude montre l'évolution de la pensée du créateur, c'est un signe qui garde vivante l'intention. Le repentir ici installe une amélioration, il dessine une belle courbe qui s'oppose au dessin précédent en jouant avec lui. Mais il le fait avec des pavés de 500 kilos chacun, 
avec une brutalité subtile qui rappelle plus la violence de la plume de Rembrandt ou les dessins des artistes modernes que les feuilles classiques de Léonard de Vinci.

Figure 6a

Caviardage

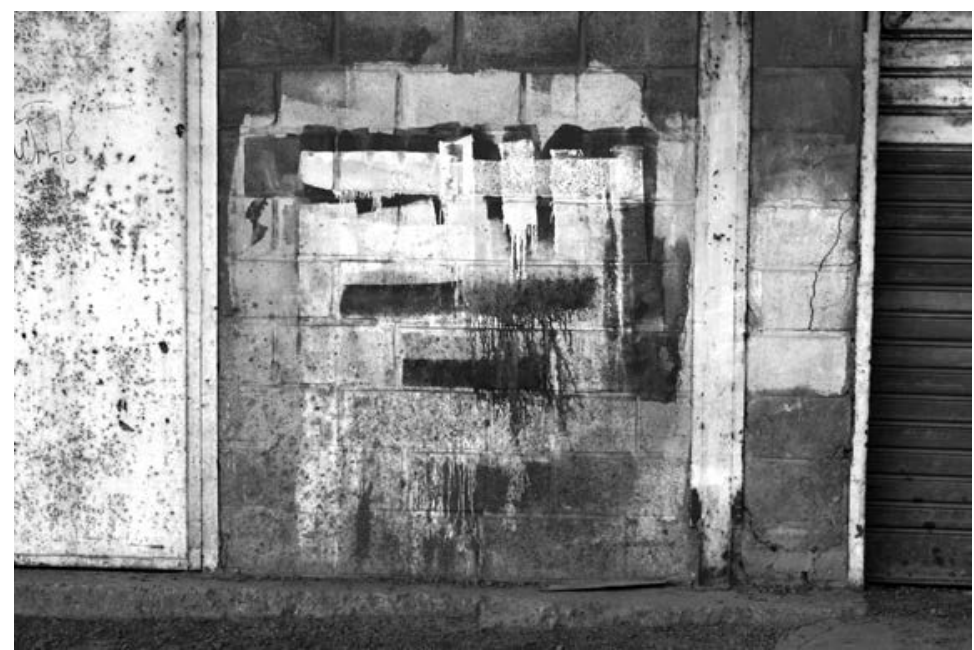

Les caviardages sont les couches de peinture recouvrant les graffitis ou les traits qui les biffent pour les rendre illisibles.

(C) Dector et Dupuy 
Figure $6 \mathrm{~b}$

Coincement

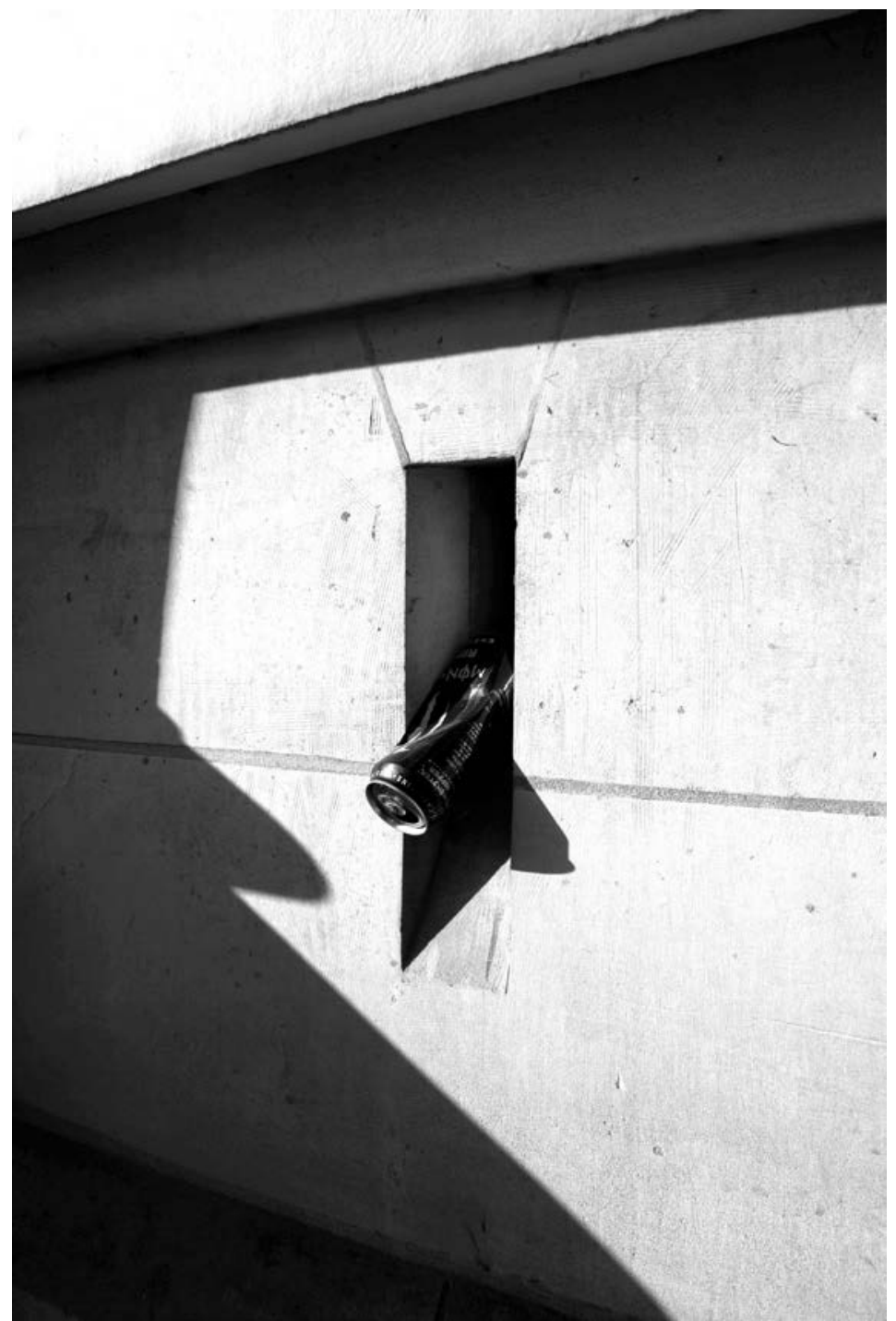

Les coincements sont des photographies d'objets coincés intentionnellement (entre les barreaux d'une grille, les branches d'un arbre, dans un grillage...).

(c) Dector et Dupuy 
Figure 6c

Dépôt

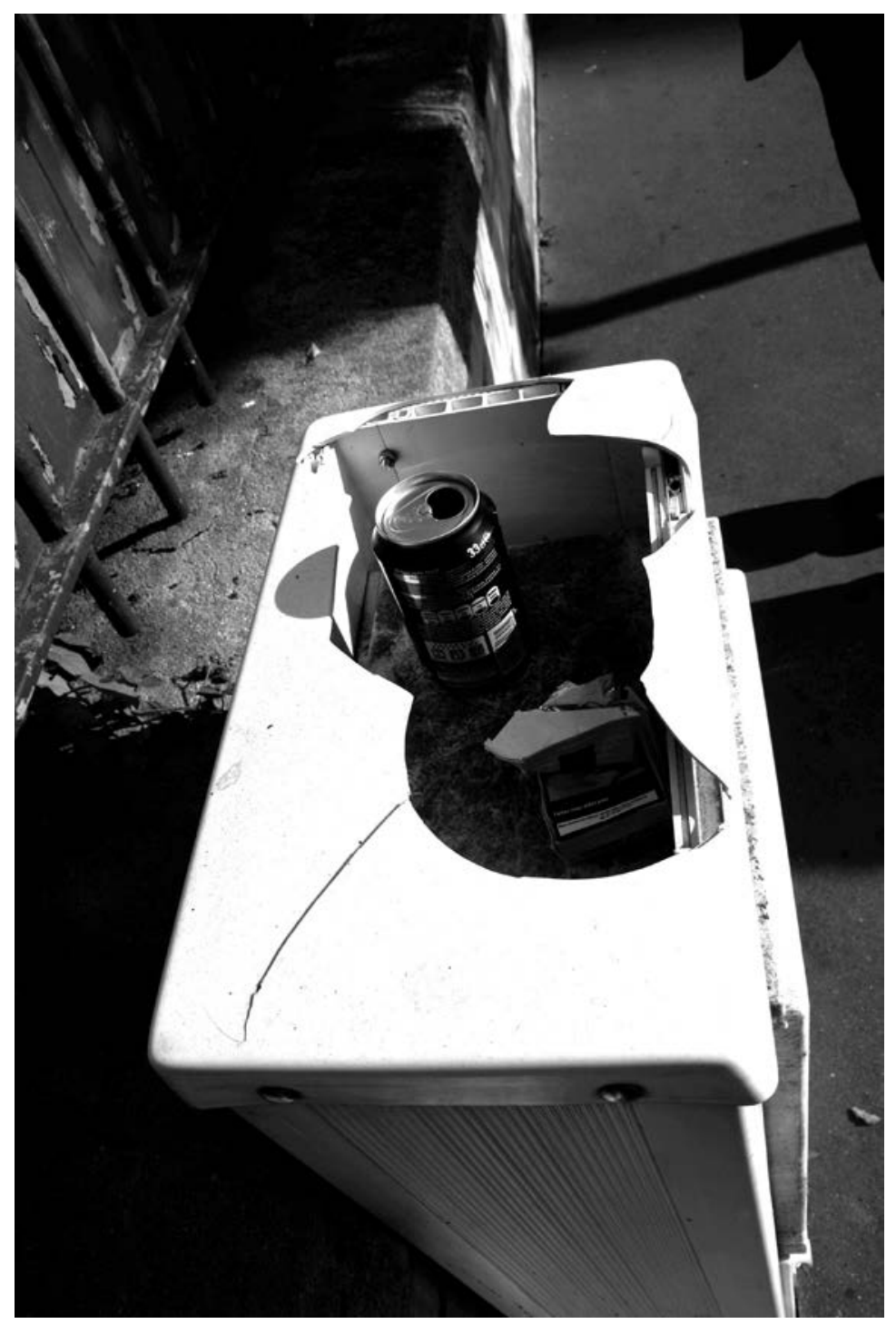

Les photographies de dépôts montrent des ordures déposées de manière originale (dans le coin d'une fenêtre, sur une sculpture, sur un piédestal...).

(C) Dector et Dupuy 
Figure 7

Repentir

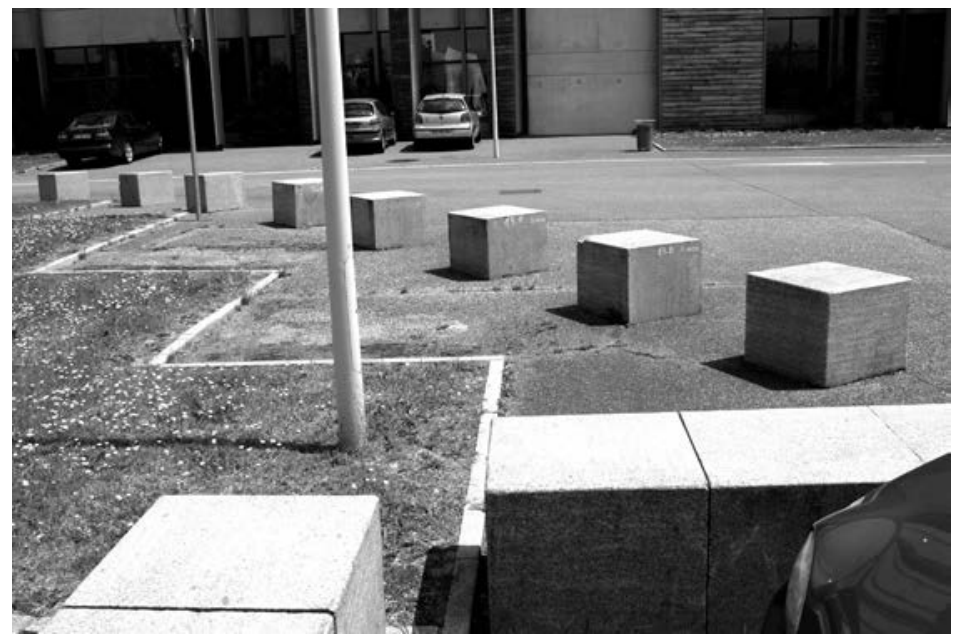

Composition abstraite sur le parking de l'ésam à Caen.

"Le repentir ici installe une amélioration, il dessine une belle courbe qui s'oppose au dessin précédent en jouant avec lui. Mais il le fait avec des pavés de $\mathbf{5 0 0}$ kilos chacun."

(c) Dector et Dupuy

La manière dont Dector et Dupuy enchantent le réel le plus trivial par la description, la narration ou la comparaison relève d'un processus d'artification. C'est une manière de rendre artistique ce qui n'était pas considéré comme tel auparavant, un changement qui prend effet dans l'esprit des spectateurs au moment de la performance. La forme même de leur action - la visite guidée - est significative de ce changement de régime ontologique: le guide commente des objets de valeur; il accompagne les phénomènes de patrimonialisation, garantissant auprès du public le prestige, la beauté, l'importance de ce qui est visité. Tout ce que les artistes élisent pour faire leur visite est donc élevé au rang de bien identitaire et culturel d'intérêt, à l'instar des œuvres d'art. Dector et Dupuy s'approprient de la sorte l'espace du MéPIC, ils y apposent une empreinte esthétique et patrimoniale, signe d'un nouvel usage de l'estuaire de l'Orne qui s'ajoute 
aux autres et préfigure la vocation artistique et touristique que l'exposition va lui attribuer ${ }^{20}$. Cette appropriation par l'art est cohérente avec l'identité de Dector et Dupuy: elle indique un vécu, un ressenti particulier; elle est la pratique d'artistes de cet espace. En ce sens, ils fabriquent un territoire, leur territoire, puisque leur performance regroupe des lieux séparés et leur confère un sens commun, une identité (tant pour les artistes que pour les spectateurs), celle de Balle perdue. Pour autant, cette sémiotisation d'un espace comme territoire d'action, presque de jeu, de Dector et Dupuy produit-elle du lien pour les artistes et pour les spectateurs? A-t-elle des répercussions sur la manière de le vivre?

La vision artistique que portent Dector et Dupuy sur la zone d'implantation du musée éclaté n'est pas dépourvue de dimension géographique. D’une part, elle s'appuie sur des aménagements urbains dont la fonction et la lisibilité se trouvent mises en défaut - un panneau de signalisation entouré de barbelé, un poteau rouillé, une infrastructure routière... Elle est donc attentive à la gestion et à la sémiotique spatiales. D'autre part, elle qualifie par défaut un type d'espace: les artistes choisissent de recourir de manière manifeste à la poétisation du réel et à sa transformation en art pour des lieux en déshérence, peu fréquentés des locaux: friche industrielle de la Presqu'île proprement dite; plateau de Colombelle avec sa nouvelle zone d'activité sans âme... L'écart extrême entre les deux bouts de la chaîne - un objet sans aucune valeur, presque destiné au rebut dans des zones blanches ${ }^{21}$ se transforme en œuvre culturelle d'intérêt - résonne à la fois comme un défi pour les artistes, qui testent ainsi la performativité de leur discours, et une amplification du processus d'artification rendu d'autant plus visible que le décalage est grand. À travers

$20 \quad$ La performance de Dector et Dupuy anticipait de quelques jours l'ouverture du MéPIC: elle s'est déroulée le 19 juin 2013 tandis que l'exposition a été inaugurée le 27 juin 2013 et s'est achevée le 27 octobre de la même année.

21 Selon les termes de Philippe Vasset dans Un livre blanc. Récit avec cartes (Paris, Fayard, 2007). Il s'agit de lieux indéterminés au sens où ils n’ont pas d'identité ou d'usage précis (à l'inverse d'une forêt, d'une route, d'une ville, d'un lotissement, d'une zone commerciale, de champs...). Ce sont les "blancs» des cartes. 
cette transfiguration, les spectateurs prennent alors conscience de l'importance de quantité de lieux méprisés dans l'expérience esthétique quotidienne ${ }^{22}$; ils construisent une première relation au territoire de Balle perdue.

\section{Du paysage vu au paysage vécu}

Prendre conscience de la possibilité de ressentir une expérience esthétique devant des objets sans qualité ou des zones sans attrait est nécessaire à la poursuite de la performance. Elle construit un objet d'art-lieu, signale la possibilité qu'un espace soit une œuvre d'art, et donc puisse avoir un effet sur le spectateur. Or, c'est précisément par cet effet sur le spectateur, qui repose sur la dimension sensible du vécu et des pratiques spatiales, que Dector et Dupuy vont engendrer et communiquer des savoirs géographiques sur la Presqu'île au sens large : Balle perdue proposait de faire l'expérience de différents types d'espaces, les six zones ayant été retenues pour leur atmosphère singulière. Leur visite cherche ainsi à provoquer une émotion esthétique, et ce, au moyen de la coprésence d'un environnement et du commentaire ou de la narration qu'en font Dector et Dupuy. Cette émotion esthétique est celle qu'eux-mêmes, en tant qu'artistes, ont éprouvé au contact des lieux, leur sensibilité artistique les rendant particulièrement réceptifs aux spécificités de leur entourage.

L'un des moyens employés par Dector et Dupuy pour dispenser ce sentiment du lieu aux spectateurs, pour en donner une transposition narrative, est d'en commenter des détails. L'entrée dans le paysage des dunes de Merville-Franceville passe de la sorte par la description de quelques éléments (voir les figures 8a et 8b) :

On aime ce lieu, on aime sa vacuité; mais on est désarmé pour en parler. Pour dire l'absence on en est réduit à user de procédés littéraires: comment mieux dire le silence qu'en évoquant l'aboiement d'un chien

$\overline{22}$ Cf. le concept d'esthétique environnementale: Nathalie Blanc (Vers une esthétique environnementale, Versailles, Éditions Quae, 2008) et Émeline Eudes ("The Romantic Geographic Society, ou comment et pourquoi marcher en Finlande en 2012). Retour sur l'œuvre d'itinérance de Jussi Kivi», dans Laurent Buffet (dir.), Itinérances. L'art en déplacement, Grenoble, De l'incidence éditeur, 2012, p. 123-142. 
au loin? On peut nommer des objets pour parler de la vacuité. Un bois mort. Une cordelette serpentine, une bouteille.

Figure $8 \mathrm{a}$ et $8 b$

Vacuité
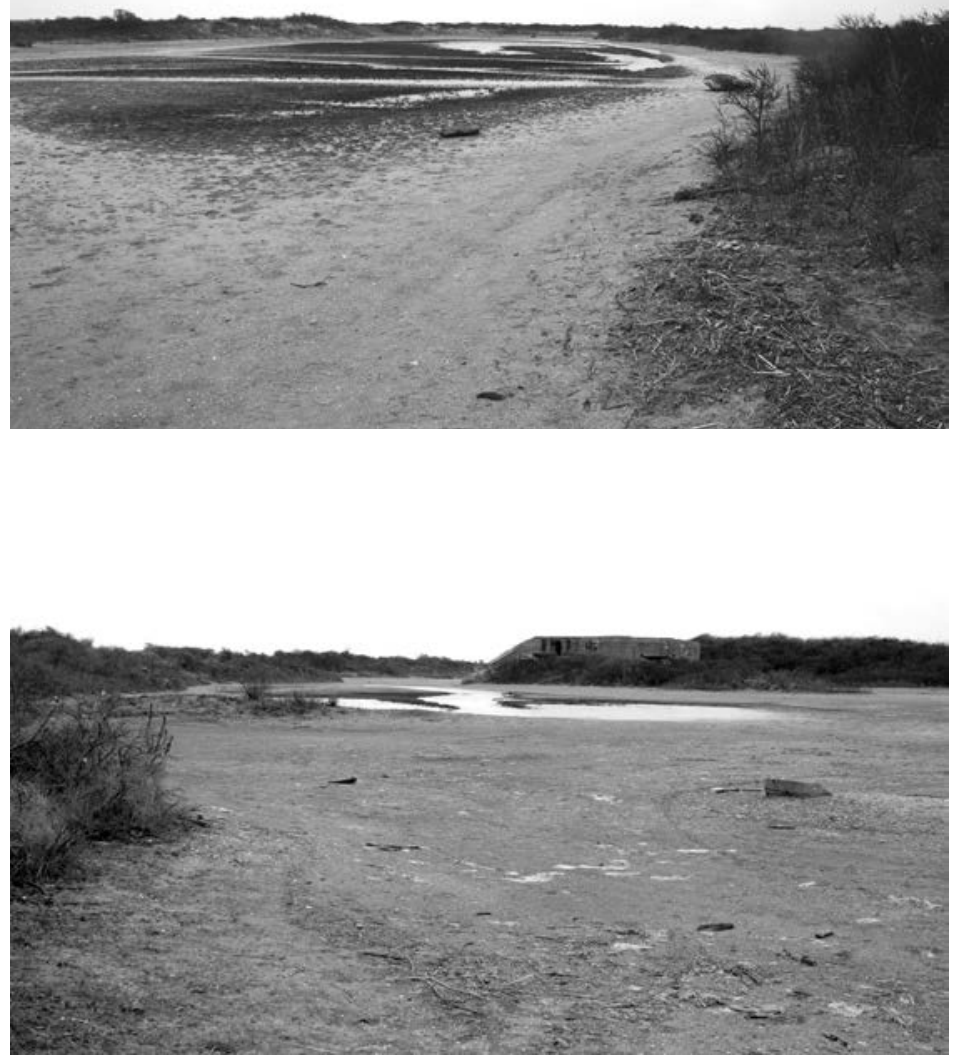

Le paysage entropique des dunes de Merville-Franceville.

"On aime ce lieu, on aime sa vacuité; mais on est désarmé pour en parler. [...] On peut nommer des objets pour parler de la vacuité. Un bois mort. Une cordelette serpentine, une bouteille."

(c) Dector et Dupuy 
Les trois objets désignés - le bois mort, la cordelette serpentine et la bouteille - conduisent progressivement le regard au loin. L'oil se perd dans le paysage comme chaque objet qui, toujours plus éloigné, se fond davantage dans l'environnement, est de moins en moins perceptible pour les spectateurs. Un peu plus loin, après avoir traversé ce vide, cette nature laissée à elle-même d'où émergent d'anciens bunkers allemands, Dector et Dupuy s'arrêtent et ramassent une balle de tennis (voir la figure 9):

On a ici deux éléments: ce bunker qui date de la guerre, mais qui a été patiné, un peu verdi par le temps que la nature a tendance à absorber. Cette sphère, pelucheuse à l'origine, qui a perdu ses poils et sa couleur, qui est verdie par le moisi, égarée dans les broussailles. Tous les deux sont dans un processus de disparition lente, ils tendent à retourner à la nature. La nature semble avoir l'intention de les engloutir. Pour l'un comme pour l'autre elle a tout son temps. Là c'est un bunker. Ici c'est quoi? On dirait que ça date d'aussi longtemps. Une balle perdue?

Figure 9

Balle perdue

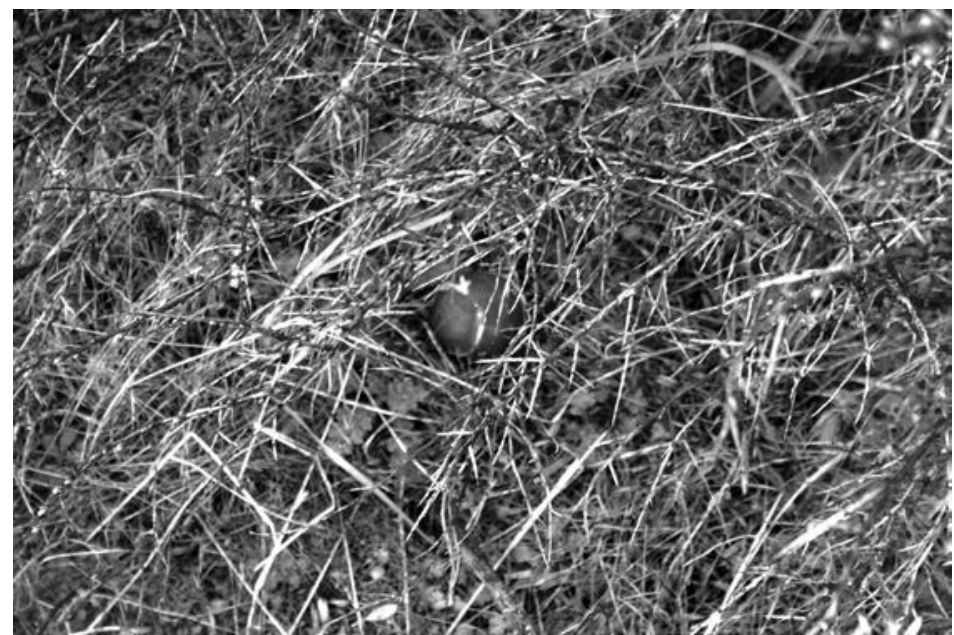

Processus de disparition d'une balle de tennis.

"Cette sphère, pelucheuse à l'origine, qui a perdu ses poils et sa couleur, qui est verdie par le moisi, égarée dans les broussailles. [...] Ici c'est quoi? On dirait que ça date d'aussi longtemps. Une balle perdue?"

(C) Dector et Dupuy 
L'équivalence que le temps et les intempéries ont produit entre ces deux objets à l'origine si dissemblables rend visible l'entropie latente sur l'ensemble du cordon dunaire de Merville-Franceville; elle fait sentir la lente action de la nature sur les choses, le retour progressif à l'état de matière indifférenciée.

Au contraire, pour exprimer le sentiment d'espace et de vide qui caractérise le Plain d'Amfréville (voir la figure 10), Dector et Dupuy engendrent la sensation inverse: ils remplissent cette gigantesque surface peuplée d'herbes folles qui entoure l'église et se trouve au milieu du village. Prenant au pied de la lettre une erreur orthographique sur la borne informative du site, ils suscitent du plein, comme ils l'annoncent: «Le fait que ce soit écrit "Plein" sur la borne informative est une erreur bien sûr mais ce n'est qu'à moitié une erreur quand on sait qu'il est arrivé au Plain d'avoir été très plein parfois". La densité des informations fournies sur l'usage de cette place au cours du temps produit tout d'abord une impression de satiété cérébrale; la mémoire des spectateurs s'encombre de données historiques énoncées sans chronologie ni liens explicites:

Amfréville accueillait jadis la plus grande foire aux animaux de la région. Pendant la révolution également le Plain a permis à de grandes manifestations à caractère révolutionnaire regroupant les communes voisines d'avoir lieu. C'était le seul endroit suffisamment vaste pour accueillir la foule. Il y avait aussi beaucoup de monde en 44 à l'inauguration du petit monument que vous voyez sur la gauche à la mémoire des commandos français et britannique et notamment celui de l'exceptionnel commandant Kieffer avec ses 177 hommes très entraînés. Aujourd'hui le Plain est rempli de grandes herbes folles avec une surface tondue de l'autre côté pendant la fête du village qui a eu lieu le samedi 8 juin dernier. 
Figure 10

Le Plain et le vide

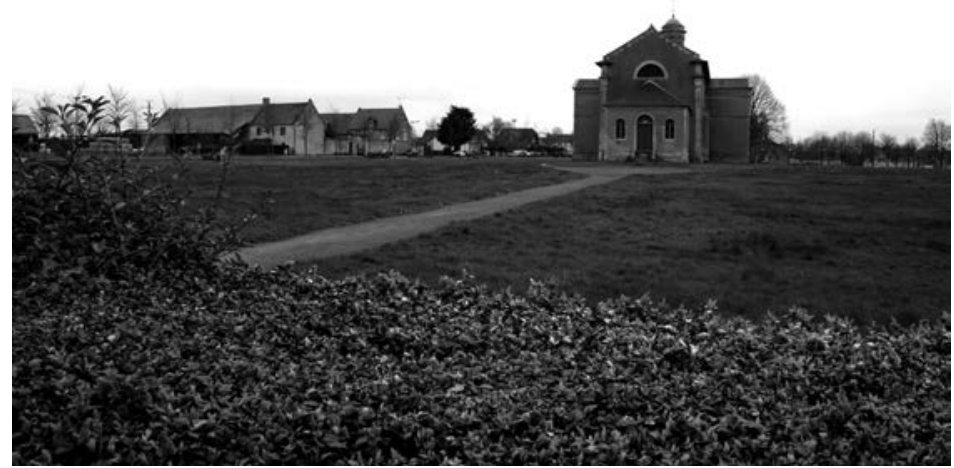

La place centrale d'Amfréville.

"Le fait que ce soit écrit "Plein" sur la borne informative est une erreur bien sûr mais ce n'est qu'à moitié une erreur quand on sait qu'il est arrivé au Plain d'avoir été très plein parfois."

(c) Dector et Dupuy

Les informations fournies sont un appel à imaginer la place remplie, frémissante, pleine de vie et de bruit, à visualiser la foule, les bêtes ou à entendre les cris, les chants. Il ne reste plus qu'à peupler physiquement cet espace, ce à quoi Dector et Dupuy s'emploient en arpentant la place avec les spectateurs. Du contraste entre ce plein créé par l'action des artistes et la vision et l'expérience du site éclot le sentiment de vide qui habite le Plain.

C'est en quelque sorte la sensation physique d'un lieu, son ambiance que les artistes tentent ainsi de faire éprouver aux visiteurs. L'arrêt dans les lotissements pavillonnaires de Bénouville (voir la figure 11) est, de ce point de vue, particulièrement révélateur: guidés par la narration, portés par les mots qui mettent l'accent sur des particularités de l'environnement, c'est 
toute la signification de ces nouvelles banlieues résidentielles que ressentent les spectateurs:

C'est un espace ouvert ici et on se sent néanmoins enfermé. [...] Dent creuse totalement bordée par des haies impeccablement taillées et opaques: sentiment d'oppression. Nous sommes comme enfermés dehors, comme dans un envers de chez soi. Le système de la haie est à son maximum! Espace comme un doigt de gant retourné: les haies enserrent et protègent les pavillons. Ici on est à l'extérieur et entouré quand même. Les haies sont des défenses: taillées comme des murs de château. [...] Qu'est-ce que cette dent creuse révèle? Que l'on est dans un village non pas de type rural mais avec une population urbaine dont l'objectif principal est d'accéder à la propriété d'une maison individuelle située à la campagne qui fonctionne de manière autarcique quant au loisir et au repos en famille. Tout est dans l'espace privé, jeu, balançoire, barbecue et rien dans l'espace public que l'on puisse partager.

Figure 11

L'envers de chez soi

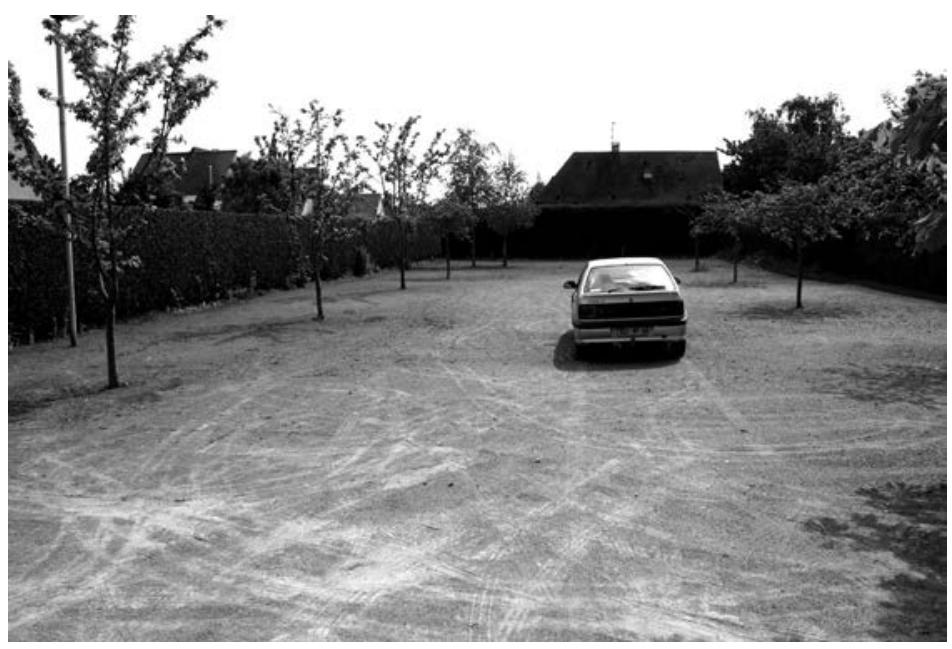

Dent creuse dans la zone pavillonnaire de Bénouville.

"C'est un espace ouvert ici et on se sent néanmoins enfermé. [...] Dent creuse totalement bordée par des haies impeccablement taillées et opaques : sentiment d'oppression. Nous sommes comme enfermés dehors, comme dans un envers de chez soi."

(c) Dector et Dupuy 
Dector et Dupuy ont beau voir dans cette dent creuse un début d'agora (elle sert lors de vide-grenier et pour les panneaux électoraux), la suite de la visite ne fait qu'accentuer la première impression. S'engageant dans un Cañon de thuya (voir la figure 12), les spectateurs, coincés entre deux hautes haies, perçoivent corporellement la sensation d'enfermement, aiguillonnés par les commentaires des artistes: "Hauteur 2,5 m au moins (debout main levée). Largeur deux bras ouverts. Proportions inquiétantes. Nouveau modulor ${ }^{23}$ ". Le regard ne peut pénétrer l'intimité des maisons, et pour limiter les nuisances et la circulation étrangère, des chicanes bloquent même l'accès de certains de ces chemins entre les résidences. Se trouve réalisé là l'idéal né au XIX ${ }^{\mathrm{e}}$ siècle d'une clôture physique totale avec l'extérieur, d'une habitation complètement étanche aux intrusions néfastes du dehors ${ }^{24}$. Le Cañon de thuya est la mesure, le modulor, d'une nouvelle ère, selon l'homophonie lancée par les artistes: «Nous sommes entrés désormais dans l'aire du thuya». Un espace et une époque où la protection contre l'extérieur est telle qu'il n'y a plus de communication, de sociabilité, mais des unités fermées juxtaposées les unes à côté des autres.

23 Il s'agit une notion architecturale de Le Corbusier: le modulor est une silhouette humaine standardisée qui sert d'étalon pour la construction de tous les bâtiments de l'architecte. Il garantit le confort de l'architecture qui est conçue selon les proportions et la morphologie humaines.

24 Philippe Ariès et Georges Duby (dir.), Histoire de la vie privée, t. 4: De la Révolution à la Grande Guerre, volume dirigé par Michelle Perrot, Paris, Seuil, 1987; Manuel Charpy, Le théâtre des objets, Espaces privés, culture matérielle et identité bourgeoise. Paris 1830-1914, thèse d'histoire contemporaine sous la direction de Jean-Luc Pinol, Tours, Université François Rabelais, 2010. 
Figure 12

Cañon de Thuya

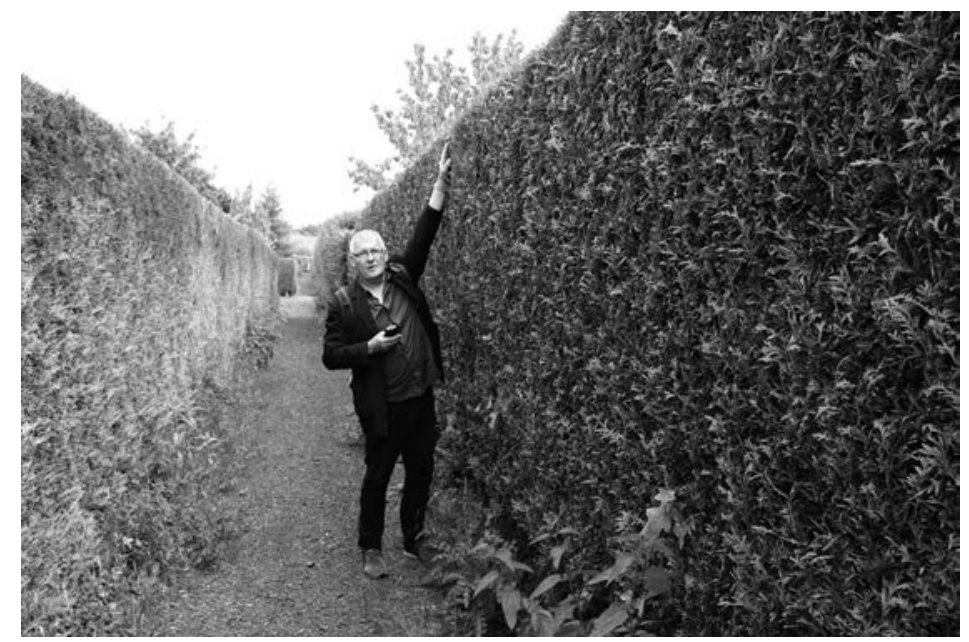

Chemin entre les haies de thuya de la zone pavillonnaire de Bénouville. "Hauteur 2,5 m au moins (debout main levée). Largeur deux bras ouverts. Proportions inquiétantes. Nouveau modulor."

(C) Dector et Dupuy

\section{Une expérience du lieu, dimensions idéelles et représentations collectives}

Dector et Dupuy convient donc indirectement les spectateurs à une expérience du lieu. Leur performance met en mots et en sensation l'imaginaire exhalé par l'espace parcouru; elle s'appuie sur la dimension esthétique de la perception spatiale pour dévoiler les significations latentes qui y sont inscrites. D'une émotion plastique, les artistes font émerger une appréciation culturelle et sociale qui rend visibles les dimensions idéelles du lieu: la toutepuissance de la nature et la fragilité humaine dans les dunes de Merville-Franceville; le repli sur soi et l'absence de vie communautaire dans les nouvelles zones résidentielles. Autrement dit, après avoir construit un territoire par le processus d'artification, ils adoptent une seconde démarche qui consiste cette fois ci, non à fabriquer du sens pour cet espace, mais à délivrer ce qui serait 
la signification intrinsèque d'un lieu via la description de manifestations extérieures "sensées traduire des intentionnalités cachées. Les comportements des acteurs sociaux [et les caractéristiques paysagères dans le cas des visites de Dector et Dupuy] peuvent se lire comme des messages, qui, à condition d'être décryptés, veulent dire quelque chose sur le territoire ${ }^{25}$ ». Bien souvent, ce sont des objets qui matérialisent cet inconscient. Attentifs aux signes, aux choses, les artistes sélectionnent ce qui fait sens dans l'environnement. Une cordelette serpentine, une balle perdue sont chargées de signifier la vacuité et l'entropie; des haies de thuya manifestent l'individualisme, redoublant de leur présence le sentiment exprimé dans le commentaire ou la description. C'est une telle approche qui se dessine sur la Pointe du Siège à Ouistreham : l'environnement traversé, les objets soulignés, les descriptions de Dector et Dupuy s'associent pour former un ensemble plastique - un objet d'art-lieu - qui donne forme et lisibilité à la sémantique de cet espace. Dans un crescendo orchestré par les artistes, la Pointe du Siège se pare d'atours qui témoignent de son urbanisme si particulier et de l'inconscient qui l'accompagne.

La narration des artistes débute par une fiction mythologique sur l'origine de la jetée de Neptune puis se poursuit par ce commentaire d'un Sentier tendre (voir la figure 13):

C'est une tendresse réciproque. Le goudron ne recouvre pas les plantes. Les plantes viennent faire un feston. Petite irruption d'herbes dans les fissures. Le tout fait un sentier tolérant, fait de respect réciproque et de limites mouvantes et irrégulières négociées en fonction des saisons. Au-delà du sentier c'est une introduction à l'harmonie du lieu fait de petites choses que l'on va parcourir et que l'on vous invite à continuer où habitation et nature sauvage coexistent sur une langue de terre entre la baie de l'Orne et la mer.

$\overline{25} \quad$ Bernard Ellisalde, op. cit., p. 196. 
Figure 13

Sentier tendre

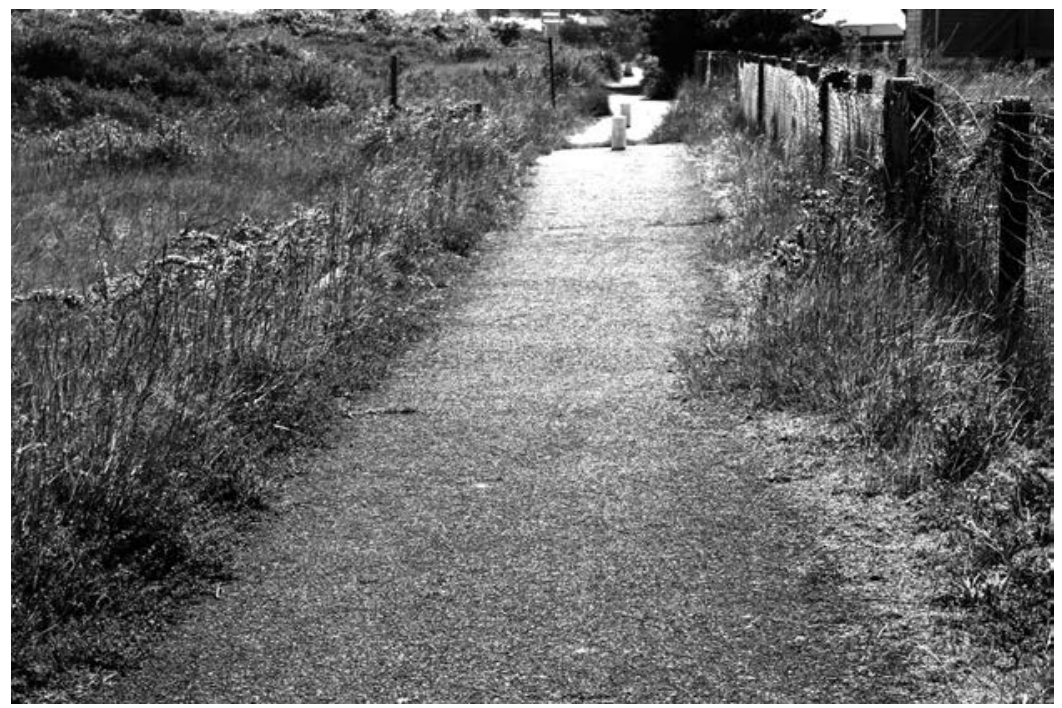

Chemin à la Pointe du Siège

"C'est une tendresse réciproque. Le goudron ne recouvre pas les plantes. Les plantes viennent faire un feston. Petite irruption d'herbes dans les fissures. Le tout fait un sentier tolérant, fait de respect réciproque."

(C) Dector et Dupuy

Cohabitation harmonieuse entre le béton et la nature, épanouissement mutuel, la Pointe du Siège se révèle, à la lecture de ce sentier, un espace préservé de la domination des hommes, c'est-à-dire des ravages du tourisme. Le lieu est en effet occupé par un espace naturel protégé et quelques habitations, des cabanes de construction précaire et d'usage saisonnier pour la plupart ${ }^{26}$. L'esprit de tolérance exprimé par le sentier est ainsi, comme le sous-entendent les artistes, celui de cet urbanisme singulier presque sauvage, à la limite de l'irrégularité pour son bâti et son occupation des sols (voir les figures 14a et 14b). Devenu insulaire avec la construction du canal de Caen à la Mer, ce cordon dunaire fossile accomplit en quelque sorte le fantasme

26 Fabienne Cosset, Ma cabane en Normandie. Chalets, petites maisons et mobile homes du bord de mer, CRéCET, Caen, 2002. 
de l'île déserte coupée du monde où il est possible de vivre en harmonie avec la nature. Il se présente comme le vestige d'un mode de vie révolu, volontiers associé à une vie communautaire et à une convivialité disparue avec les temps modernes.

Figure $14 \mathrm{a}$ et $14 \mathrm{~b}$

Cabanes sur la Pointe du siège, un urbanisme presque sauvage

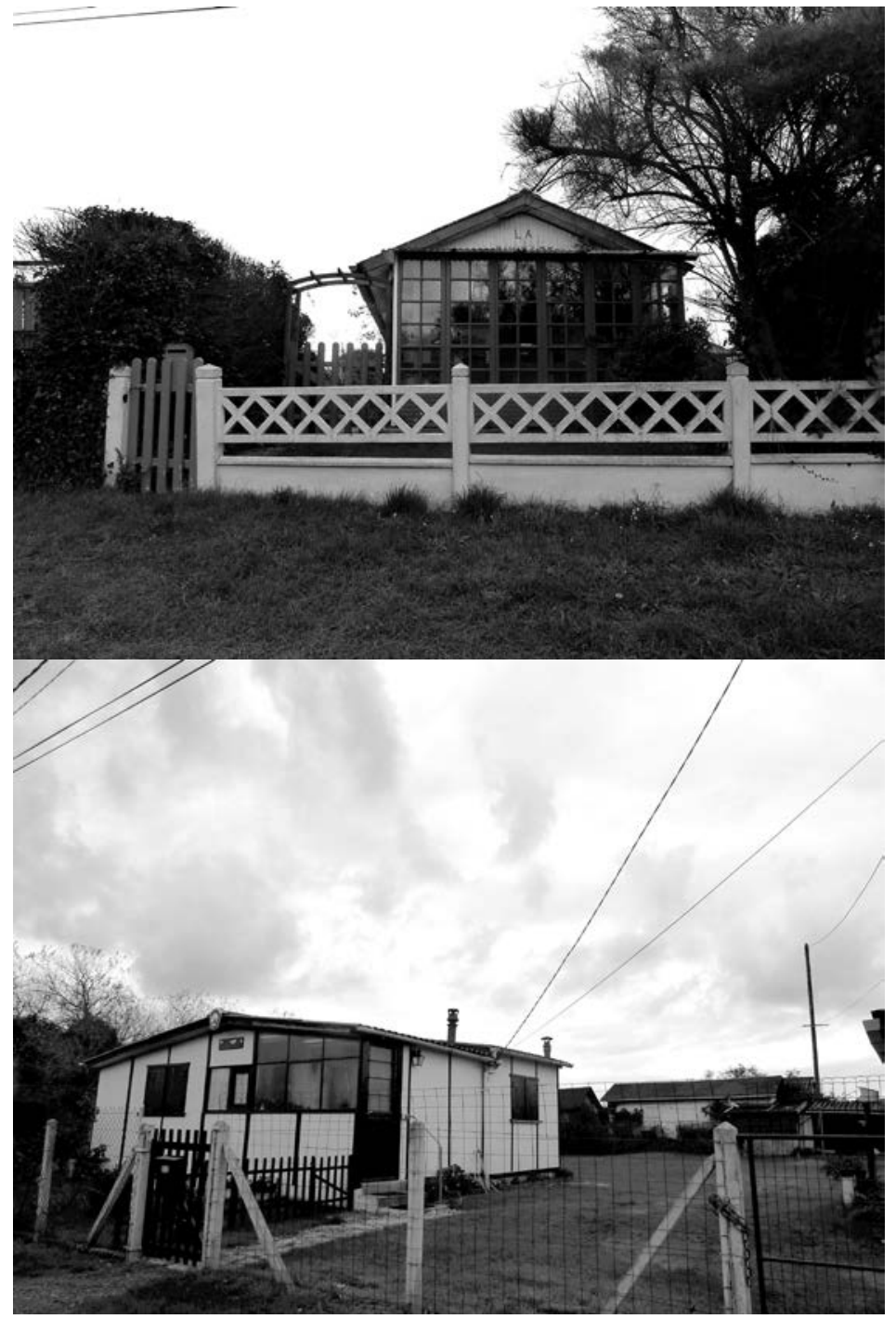

(C) Claire Le Thomas 
Dector et Dupuy approfondissent ensuite cette impression première en décrivant des objets qui incarnent à leurs yeux l'imaginaire sous-jacent du lieu - vacances, plaisir, tolérance. Chaque arrêt met l'accent sur une dimension idéelle du site pour la rendre perceptible aux visiteurs. Une sculpture exprime par exemple le renversement des valeurs qui semble à l'œuvre sur la Pointe du Siège (voir la figure 15).

Cela ne représente rien nous a dit le propriétaire. C'est seulement une souche inversée. Il nous a dit qu'il a retiré une souche pour faire sa maison mais au lieu de la jeter, il l'a plantée dans le sol devant sa maison - à l'envers. Et il l'a mise en valeur en l'entourant de pierres, de bois flottés et de galets venant de la plage. En entourant la souche de galets et de bois flotté, il a réuni la terre et la mer. Et en plantant la souche, le tronc dans la terre et les racines en l'air, il a inversé le ciel et la terre.

Figure 15

La sculpture qui ne représente rien

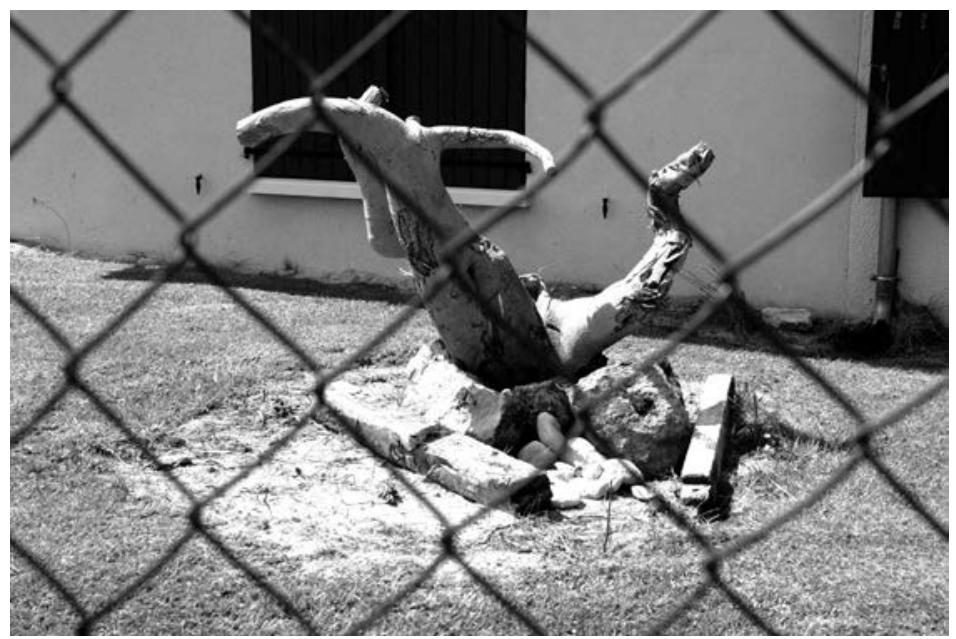

Souche renversée sur la Pointe du Siège.

"Cela ne représente rien nous a dit le propriétaire. C'est seulement une souche inversée. [...] En entourant la souche de galets et de bois flotté, il a réuni la terre et la mer. Et en plantant la souche, le tronc dans la terre et les racines en l'air, il a inversé le ciel et la terre."

(c) Dector et Dupuy 
L'analyse des artistes explicite le sens caché de cette sculpture: réunion de la terre, de la mer et du ciel, elle symbolise la liberté du lieu et son unicité. D'une part, le propriétaire a su déceler la beauté d'un objet gênant destiné au rebut et l'a transformé en œuvre d'art; d'autre part, sa morphologie tête-bêche et ses composantes (souche, cailloux, bois flottés) en font une discrète apologie de la mixité et de la subversion contre l'ordre établi. Autrement dit, elle s'accorde avec l'urbanisme exceptionnel de la Pointe du Siège dont les cabanes inversent la tendance au confortable et ne respectent pas les normes de construction actuelles mais dont la précarité autorise justement une harmonie entre l'homme et la nature; la créativité dont elle fait montre est le résultat de l'esprit d'indépendance et d'indiscipline de ce bout de terre. Son auteur n'est d'ailleurs pas le seul créateur spontané du lieu, un autre habitant s'est révélé philosophe et artiste conceptuel sans le savoir (voir la figure 16): "Le nom "Phare", l'image du Phare et là-bas le phare réel. 3 manières d'exister pour le phare. Il ne manque peut-être que la définition qui est dans le Larousse : "tour élevée portant un puissant foyer de lumière pour guider les navires la nuit" ». La référence au travail de Joseph Kosuth $^{27}$ vient entériner le caractère singulier du lieu: elle crée une équivalence entre l'unicité de l'œuvre d'art et celle de la Pointe du Siège; elle met en exergue la rareté de cet espace tant pour sa morphologie que pour son atmosphère.

$27 \quad$ Plus précisément à l'œuvre One and Three Chairs (1965, chaise en bois et 2 photographies, $200 \times 271 \times 44 \mathrm{~cm}$ ) dont un exemplaire est conservé au Museum of Modern Art de New York. 
Figure 16

Les 3 phares

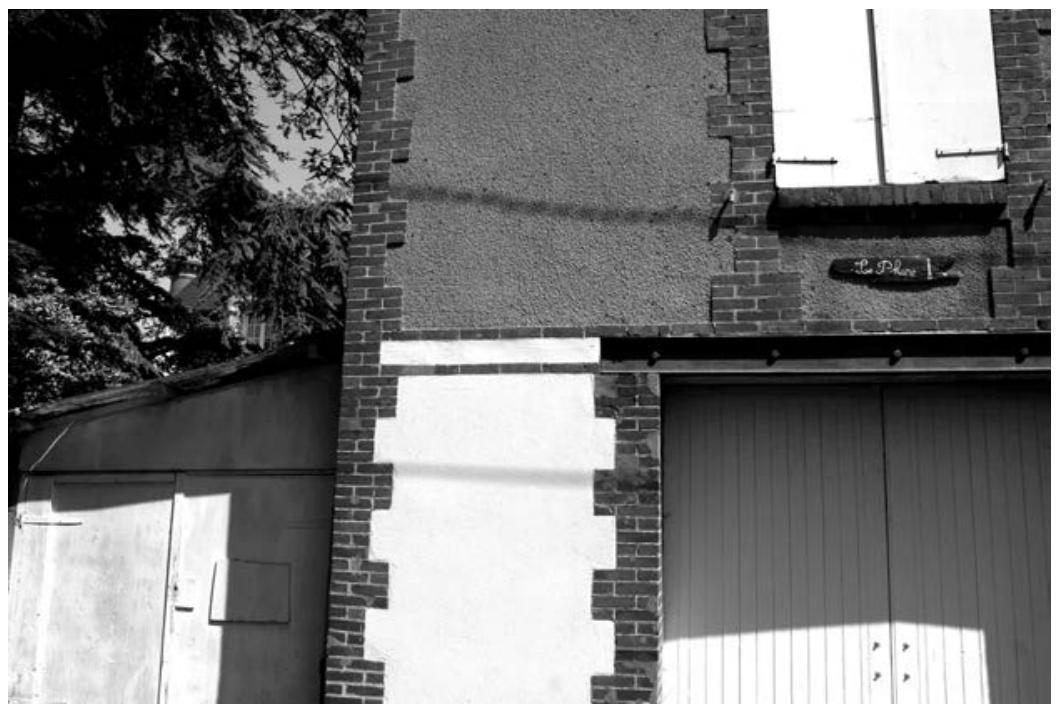

Kosuth revisité sur la Pointe du Siège.

"Le nom "Phare", l'image du Phare et là-bas le phare réel. 3 manières d'exister pour le phare. II ne manque peut-être que la définition qui est dans le Larousse."

\section{(c) Dector et Dupuy}

L'expérience que proposent les artistes à la Pointe du Siège relève d'une mise en lumière de l'inconscient du lieu; ils offrent un nouveau régime de visibilité, via la narration, le choix d'objetssignes et le parcours effectué avec les visiteurs, aux significations latentes de cet espace singulier. Ainsi, Dector et Dupuy, par leur performance, initient les visiteurs aux particularités urbanistiques et paysagères de la Presqu'île au sens large. À partir d'un espace vécu - par les spectateurs mais selon la perception des artistes -, ils accèdent à l'espace des représentations attachées aux lieux parcourus, à ces idéologies du moment qui conduisent à voir un aspect particulier de l'environnement et déterminent son aménagement. Friches, zones naturelles préservées, résidences périurbaines et leurs enjeux - recherche de nouvelles vocations, pression touristique et foncière, étalement urbain et disparition 
des sociabilités villageoises - sont évoqués via une œuvre d'art et au moyen de procédés plastiques. Balle perdue transmet des savoirs géographiques, donne à voir les mouvements qui traversent l'espace où se déploie le MéPIC ou, plus exactement, selon l'acception de Maryvonne Le Berre, les pratiques territoriales (de vie, de gestion, d'aménagement) : «l'ensemble des actions que le groupe entreprend pour assurer sa vie et son maintien sur son territoire [...] [et qui] ont pour résultat de mettre en relation les lieux qui constituent son territoire ${ }^{28}$ ».

\section{Mémoire et traumatisme: histoires de lieux}

La visite de Dector et Dupuy procède donc à une triple fabrique territoriale. Elle propose d'abord une sémiotisation unique - sous la forme d'un construit artistique - de la zone d'implantation du musée éclaté; elle crée ensuite du lien, du vécu au moyen de l'expérience esthétique; elle rend compte enfin des systèmes de représentation qui guident les interventions humaines sur cet espace. Elle s'appuie sur le subjectif, la vision et l'action de ces artistes, pour donner à voir le collectif qui travaille les lieux. Dector et Dupuy mêlent ainsi leur regard à celui des habitants; ils abordent par la bande leur ressenti, un ressenti qui s'énonce au présent mais se construit aussi sur un passé commun. Cette dimension historique du territoire est explorée par les artistes au moyen de lieux ou d'objets symboliques de l'histoire de l'Estuaire. Ils dévoilent alors des traumatismes dont l'impact physique équivaut celui laissé dans les mémoires. Avec cette double mise en perspective, ils extraient l'expérience spatiale de l'individualité et du présent qui la caractérisent - l'appréhension de l'environnement s'effectue toujours dans un ici et un maintenant propre à chacun - pour lui superposer la temporalité géographique du paysage et la multiplicité du territoire.

Larticulation du temps et de l'espace, la présence du passé dans le présent que décèlent Dector et Dupuy est d'abord inscrite dans l'espace, c'est-à-dire qu'elle se signale sous la forme de traces

28 Régis Keerle et Raymonde Séchet, op. cit., p. 9. 
matérielles que les artistes découvrent et commentent, à l'instar des trous du phare de Ouistreham (voir la figure 17).

Quand on touche ce granit de la main on prend conscience de la fragilité, de la vulnérabilité de notre chair. Et si on voit les impacts de balles qui ont fait sauter ces gros éclats sur ce même granit on mesure la vitesse, la violence des impacts des balles de différents calibres. On imagine très bien les dégâts que doit pouvoir faire une balle dans notre corps. La guerre, dit Jean-Luc Godard, c'est « faire entrer des morceaux de fer dans des morceaux de chair». Ce phare n'est pas répertorié comme un monument de la guerre de 39/45. Et pourtant c'est peut-être le plus poignant. Ce pourrait être un Monument aux blessures.

Figure 17

Le fer et la chair

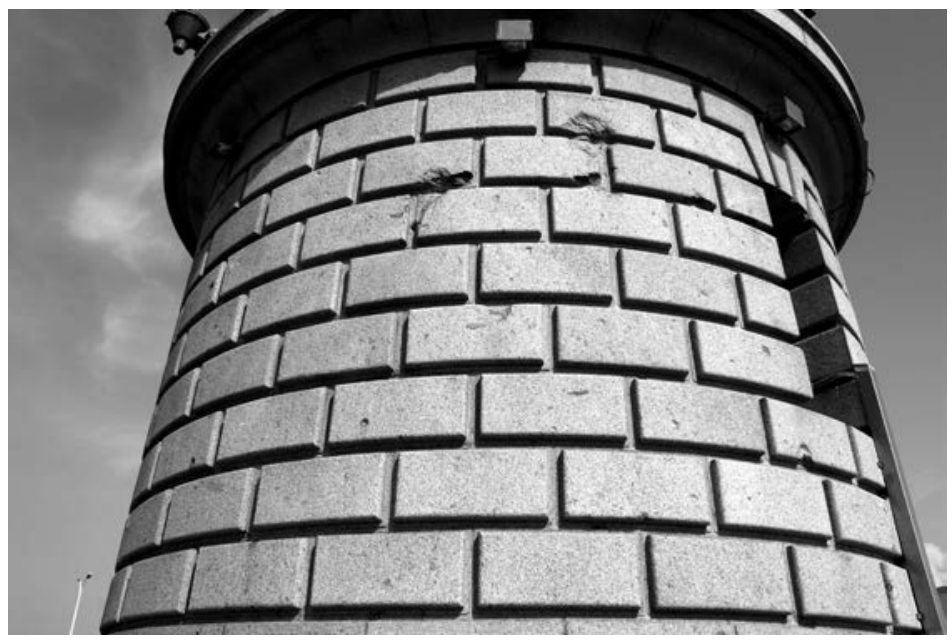

Impacts de balles sur le phare de Ouistreham

"Quand on touche ce granit de la main on prend conscience de la fragilité, de la vulnérabilité de notre chair. [...] Ce phare n'est pas répertorié comme un monument de la guerre de $39 / 45$. Et pourtant c'est peut-être le plus poignant. Ce pourrait être un Monument aux blessures."

(c) Dector et Dupuy

Discrètement, Dector et Dupuy relèvent le poids de la Seconde Guerre mondiale et du débarquement sur la région. Au lieu de s'attacher à un des nombreux mémoriaux du secteur, qui ajoutent de nouvelles marques à un espace déjà couvert de cicatrices, ou 
de décrire un vestige de l'époque pour évoquer cet épisode, leur regard se porte sur un stigmate peu visible et pourtant emblématique du rapport du Calvados à cette période. Emblématique car les impacts de balles sur le granit sont rapportés par la narration à une expérience corporelle, c'est-à-dire personnelle, intime qui, en faisant appel à notre conscience physique, joue sur l'affect et exacerbe les sentiments. Le trouble ressenti par les spectateurs permet alors d'approcher la passion avec laquelle la Seconde Guerre est encore vécue par une partie de la population et les émotions qu'elle cristallise. Rattacher les empreintes spatiales de la guerre à l'intime, c'est aussi rappeler que ces traces ne sont rien sans l'humain qui leur donne sens, que ce sont les habitants qui font le territoire. Emblématique ensuite car le choix de remémorer cet événement via des résidus indiciels souligne l'ampleur du traumatisme dont les ramifications se déploient jusque dans des lieux de prime abord étrangers à la guerre. Emblématique enfin car en évitant le monument et le site historique, Dector et Dupuy traitent de la guerre et du débarquement de biais, manifestant la relation complexe qu'entretient la région avec cette période, les élus désirant estomper cet épisode qui prend trop de place dans les représentations de la BasseNormandie. La participation de la région au Festival Normandie Impressionniste et l'élaboration du musée éclaté ont en effet été dirigées par une volonté politique d'attractivité territoriale: il s'agissait entre autres de remédier au déficit de représentation de l'agglomération caennaise et de diversifier son offre touristique jusqu'à présent essentiellement tournée vers le tourisme de guerre avec le mémorial de Caen et les plages du débarquement ${ }^{29}$.

29 Le MéPIC a en effet été organisé dans le cadre du second Festival Normandie Impressionniste. Il a obtenu le soutien des élus - pour ne pas dire qu'il a été commandé par Philippe Duron, maire de Caen et président de la communauté d'agglomération Caen-la-Mer, qui souhaitait que la ville et la BasseNormandie se démarquent par une grande manifestation d'art contemporain. Le musée éclaté s'inscrit ainsi au cœur de la politique culturelle mise en œuvre sous son mandat afin d'améliorer l'image de l'agglomération caennaise. Les projets de réaménagement des waterfront de la Presqu'île au sens strict jouent un double rôle dans cette recherche d'attractivité territoriale: en tant que foncier disponible où localiser des institutions culturelles phares d'une part; 
Dector et Dupuy, malgré l'omniprésence de ce passé dont les vestiges émaillent la région, ont respecté l'esprit qui a présidé à l'organisation du Second Festival Normandie Impressionniste en Basse-Normandie et du musée éclaté: sans faire l'impasse sur cette mémoire, ils ne l'ont abordé, sur une visite comportant une trentaine d'arrêts, qu'à deux reprises et à chaque fois pour des traces peu visibles sans possibilité de valorisation touristique mais susceptibles de provoquer un fort investissement émotionnel sous l'effet du commentaire des artistes.

Dector et Dupuy ont exploré un autre passé ayant laissé son empreinte sur la Basse-Normandie, celui d'une désindustrialisation récente et spectaculaire qui a conféré à l'agglomération caennaise l'image d'un territoire sinistré. La fermeture de la Société métallurgique de Normandie (SMN), au milieu des affrontements et des grèves, en 1993, et le ralentissement progressif du trafic du port ${ }^{30}$ - deux univers à forte identité et donc à grande visibilité - ont en particulier marqué les esprits ${ }^{31}$ : la disparition de ces activités a généré deux vastes friches, la Presqu'île au sens strict et le plateau de Colombelles, qui sont le rappel douloureux et amer de la fin d'une époque glorieuse et prospère. La mesure du traumatisme était particulièrement flagrante dans le discours de Marc Pottier, maire-adjoint à Colombelles en charge de la culture et directeur de l'Agence régionale de Conservation de l'Image et du Son de Basse-

en tant que moyen de développer les usages récréatifs et balnéaires de l'eau d'autre part. Voir à ce sujet : Aucame, «La place du tourisme normand en France» (Qu'en savons-nous?, n 34, mai 2011, 4 p.); Caen, Conseil municipal de Caen, Caen, La CULTURE en capitales. Vivre et sémerveiller ensemble (communication sur le projet culturel de la Ville de Caen présentée au conseil municipal du 14 septembre 2009, [en ligne] http://www.caen.fr/culture/ projetCulturel//; site consulté le consulté en septembre 2014); Adèle Fleury, "Comment la ville de Caen peut-elle pérenniser son tourisme patrimonial tout en diversifiant son offre touristique afin de créer une réelle destination touristique?» (mémoire de master 2 en tourisme, Paris, Université de Paris 1, 2012) et Claire Le Thomas, "Art de l'entre-deux...» (loc. cit.). Aucame, "Le port de Caen-Ouistreham", Qu'en savons-nous?, n 8, octobre 2008, 4 p.

31 La fermeture des usines Moulinex à Alençon dans l'Orne en 2001 contribue aussi à cette image d'une région sinistrée. 
Normandie (ARCIS) rencontré lors des repérages. Dans cet entretien de plus d'une heure, il n'a eu de cesse de rappeler le passé glorieux de la ville et ses environs: Caen, capitale du royaume anglo-normand au Moyen Âge dont la pierre a servi à construire des monuments emblématiques de l'Angleterre (la tour de Londres, Windsor); Caen, troisième port français dans les années 1950-60 et pionnier avec la SMN dans l'aménagement de complexes sidérurgiques sur l'eau ${ }^{32}$; la SMN, usine à la pointe de la technologie et ses 7900 employés... Pourtant, ou précisément en réponse à cette blessure, les projets de réaménagement de la Presqu'île et du plateau de Colombelles tendent à effacer ce passé sans effectuer de véritable travail de mémoire ${ }^{33}$. Ils sont animés par une volonté d'aller de l'avant et de tirer un trait sur une réalité économique et sociale pénible, envisageant ces terrains comme du foncier disponible à valoriser pour sa proximité avec le centre-ville, son rapport à l'eau ou sa surface exploitable ${ }^{34}$.

$A$ contrario de cette manière de penser le territoire par les aménageurs et le politique comme un espace vierge à réinventer pour l'avenir, comme une zone inerte qui ne serait le support

$\overline{32}$ Qui préfigure la «japonification sur site» et les complexes industrialo-portuaires qui se développent à partir des années 1960. Il s'agit de zones qui combinent sur un même espace des activités industrielles et portuaires. Les entreprises qui s'y installent exploitent les ressources locales et bénéficient de la proximité d'un port pour importer et exporter matières premières et marchandises. Ces zones ont ainsi un rôle d'interface entre une région et le reste du monde. La SMN, spécialisée dans la fabrication de l'acier et sa transformation, profitait des gisements de fer de la région (mine de Soumont) et du canal de Caen à la Mer pour recevoir le charbon nécessaire au fonctionnement des hauts-fourneaux et expédier ensuite une partie de sa production par voie maritime.

33 Il faut toutefois préciser que la ville de Colombelles a effectué plusieurs actions dans ce sens, notamment par le biais de résidences artistiques sur le Plateau. Un projet de transformation de la grande halle de la SMN en pôle culturel a également échoué. Néanmoins, il n’y a pas de réelle exploitation mémorielle et culturelle des vestiges de ces deux univers ouvriers, de projet global qui prendrait en compte et valoriserait les restes architecturaux des activités sidérurgiques et portuaires à l'image de l'île de Nantes ou du bassin de la Ruhr.

34 Le nouveau quartier des Rives de l'Orne, construit sur les friches adjacentes à la Presqu'île proprement dite, est emblématique de cette manière de penser. 
d'aucune dynamique et nécessiterait une intervention extérieure pour exister, Dector et Dupuy se sont attachés à faire surgir les souvenirs enfouis et les fantômes du passé, c'est-à-dire à animer les lieux pour lever le voile sur les multiples mouvements qui les ont modifiés et les modèlent toujours. Ils opposent alors le territoire du projet à celui des habitants. Sur le plateau de Colombelles, c'est toute l'histoire de la SMN qu'ils sollicitent en racontant un épisode peu connu dévoilé par Marc Pottier:

Entre la Chine et la France, il y a eu des allers et retours assez intéressants qu'une anecdote vient rassembler de manière étonnante: après la Première Guerre mondiale, la France a créé un programme d'invitation d'étudiants chinois pour remercier la Chine de son aide durant la guerre. L'un de ces étudiants est venu étudier à Caen où il a rencontré l'immigration chinoise qui travaillait dans la SMN. Devant la misère de la condition ouvrière, il a décidé d'entrer au Parti Communiste Chinois et a entamé une carrière politique. Cet étudiant s'appelait Deng Xiaoping, successeur de Mao Tse Toung, le "petit timonier». Celui-là même qui plus tard - ironie de l'histoire - a décidé d’ouvrir la Chine aux marchés capitalistes.

La SMN est en effet née pendant la Première Guerre mondiale de la nationalisation de la société des hauts-fourneaux de Caen fondée par l'industriel allemand August Thyssen ${ }^{35}$. Fleuron de la sidérurgie française, la Société normande de métallurgie, qui deviendra la Société métallurgique de Normandie vers 1924-25, fait la richesse et la fierté de la région pendant l'entre-deuxguerres et les Trente glorieuses ${ }^{36}$. L'anecdote, qui fait du séjour caennais de Deng Xiaoping l'origine de son ambition et de sa carrière politiques, traduit et accentue tout à la fois l'importance historique de l'usine et la notoriété qu'en tire Caen. Paradoxalement elle évoque également ce qui a causé sa fermeture en 1993: c'est l'ouverture aux marchés capitalistes réalisée par Deng Xiaoping qui a précipité la fin de la SMN, celle-ci ayant été délocalisée en Chine - une partie de l'usine a même été achetée pour être

35 Elle fait suite à la mise sous séquestre des biens allemands.

36 Elle s'accompagne également de la construction d'une importante cité ouvrière aux équipements multiples (école, dispensaire, théâtre, bibliothèque, coopérative...). Cf. Aucame, "La cité plateau. Une cité-jardin issu du paternalisme industriel», Qu'en savons-nous?, n 14, juillet 2009. 
remontée là-bas. Mise en scène et narrée par les artistes en face des deux vestiges témoignant de ce passé industriel - le réfrigérant et la grande halle (voir la figure 18) - et après avoir traversé une partie des 300 hectares qu'occupait la SMN, l'anecdote donne un sens à cet espace étrange, immense terrain vague progressivement repeuplé par des sociétés agroalimentaires et tertiaires. Elle prend valeur de parabole, condensant l'histoire du site tout en faisant émerger la mémoire refoulée du lieu - tant la gloire passée que la brutalité de la fermeture et du démantèlement. Le récit allégorique de Dector et Dupuy se présente alors, à l'instar des autres souvenirs cachés mis au jour, comme une initiation aux couches temporelles et relationnelles du territoire, à la dimension historique et sociale du géographique, seules capables de donner vie aux espaces en dévoilant le tissu de relations qui unissent les hommes aux lieux.

Les artistes transforment avec leur narration un objet du passé en objet de passé, c'est-à-dire un espace perçu comme obsolète et appartenant à une époque révolue, en support de mémoire, de récits qui irriguent le présent, dont le souvenir a une portée contemporaine. Ils vont ainsi à l'encontre de la tendance générale à l'oubli et affirment par la même occasion l'importance de l'histoire pour inventer de nouveaux usages aux lieux, la prise en compte du passé et des relations affectives nouées avec les habitants facilitant la greffe. Plus généralement, en examinant le passé des lieux, ils poursuivent leur peinture de la Presqu'île au sens large et font remonter à la surface deux refoulés ${ }^{37}$ constitutifs de son identité: le débarquement et les activités industrialo-portuaires.

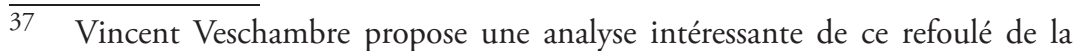
mémoire ouvrière caennaise. Il voit cet effacement comme le signe d'un rapport de classe et de domination. S’il étudie essentiellement le cas de la SMN, cette interprétation vaut aussi pour le passé portuaire de la ville (Vincent Veschambre, Traces et mémoires urbaines: enjeux sociaux de la patrimonialisation et de la démolition, Rennes, Presses universitaires de Rennes, 2008. 
Figure 18

Rondes

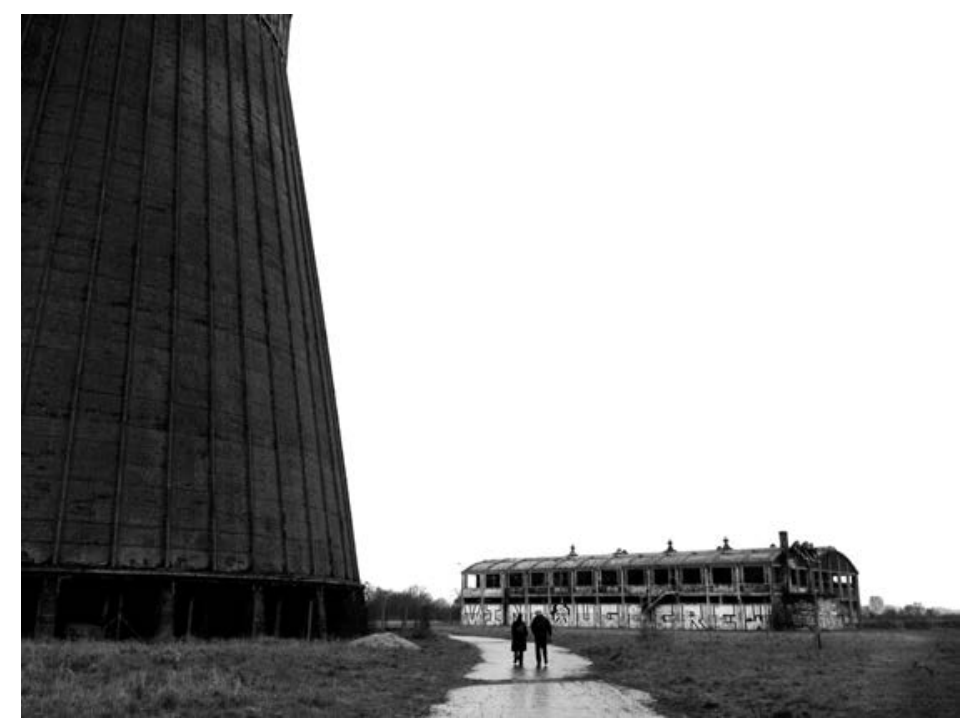

Le réfrigérant et la grande halle sur le plateau de Colombelles, vestiges de la SMN.

(C) Dector et Dupuy

\section{Conclusion}

Balle perdue, tout en étant l'expression du regard de Dector et Dupuy et le reflet de leur pratique de l'espace d'implantation du MéPIC, s'est montrée capable de prodiguer des savoirs géographiques sur cette zone, savoirs qui contribuent à la caractériser et pourraient la constituer comme un territoire. Ils ont mis au jour les liens qui unissent les habitants à certains lieux et participent à leur identité : le rapport au balnéaire et à l'eau, le développement de la dynamique résidentielle et périurbaine, la prégnance d'un double passé - débarquement et désindustrialisation. Il ne manque à cet inventaire que la permanence de l'affectation portuaire. De toute la culture maritime présente dans l'Estuaire, les artistes ne mentionnent rapidement que la 
maison des lamaneurs ${ }^{38}$ dans le square Aristide Briand à Ouistreham. Cette absence est néanmoins significative de la place problématique du port dans la ville et les communes avoisinantes: alors qu'il est toujours en activité et participe à l'économie de la région, il est invisible aux yeux de la majorité de la population. Le bassin Saint Pierre devenu port de plaisance, les bateaux marchands ne mouillent plus dans la ville et le port de commerce s'est déplacé en aval dans une zone peu fréquentée; le trafic est donc peu apparent, et ce, d'autant plus que l'accès aux quais est restreint aux acteurs portuaires.

S’ils ont dégagé des représentations collectives et des identités qui s'incarnent dans des lieux, leur performance a-t-elle pour autant réussi à les mettre en relation? Pour les spectateurs sans doute, mais sur la base d'un lien fragile et peu courant dans les approches du territoire : celui de l'art et du sentiment esthétique. Le caractère éphémère et restreint de ce liant - sans inscription durable, il est en quelque sorte limité au temps et au public de la performance, même s'il peut avoir des prolongements dans l'esprit des spectateurs et toucher de cette manière une audience plus large - réduit sa propension à fabriquer de la territorialité pour les habitants. L'absence, pour l'instant, de réelle existence politique et administrative pour cet espace, ne permet pas d'articuler ces territoires produits par le bas et approchés par Dector et Dupuy avec le territoire du projet façonné d'en haut dont le musée éclaté délimitait l'espace. En revanche, pour les artistes et les spectateurs il y a bien création d'un territoire, celui de Balle perdue. La performance de Dector et Dupuy regroupe sous un regard commun - celui des artistes, qui est aussi celui de l'auditoire puisque le principe de leurs visites est justement de partager cette vision, de la faire adopter aux spectateurs - les quinze communes du MéPIC. Elle est une appropriation et une sémiotisation d'un espace (qui ne se superpose d'ailleurs pas totalement à celui du musée éclaté), par le biais d'une expérience collective, d'un vécu ensemble. Aussi, la dimension performative de Balle perdue consiste dans cette modification la perception habituelle

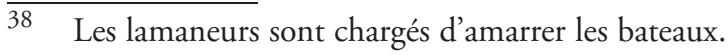


de la Presqu'île au sens large via une fabrique territoriale : ce qui apparaissait comme des lieux séparés devient un ensemble unifié par le regard et l'expérience artistiques; de cet ensemble naît la conscience, pour un auditeur sensibilisé aux problématiques touchant cet espace, de son passé, de son présent et de son avenir commun en tant qu'agglomération de Caen ${ }^{39}$. Ce changement de perception est aussi celui, pour chaque lieu, de la mise en lumière de ses dimensions idéelles et sociales : de belles résidences pavillonnaires deviennent des symboles de l'individualisme et de la périurbanisation, des dunes se transforment en emblèmes des forces entropiques de la nature, le Plateau se change en école de formation politique...

Il s'agit finalement de dépasser notre appréhension subjective des lieux pour lui substituer un regard géographique, c'est-à-dire attentif aux rapports des hommes à leur environnement, aux usages qu'ils en font ou à l'évolution générale des manières de penser, d'administrer et de vivre les espaces. Balle perdue se présente alors comme une sorte d'immersion au sein du territoire d'observation du chercheur, la démarche de Dector et Dupuy étant assez proche de celui-ci : comme le géographe, Dector et Dupuy étudient un espace socialisé dont ils tentent de révéler l'organisation, le fonctionnement, les représentations, les acteurs et leurs actions, voire la territorialité. Puisque la performativité de leur visite revient, entre autres, à fabriquer un territoire, les artistes interrogent indirectement le caractère construit de la territorialité, qui pourrait n'être que l'expression du point de vue du chercheur. C'est donc toute la complexité de la notion de territoire que révèle en dernière instance l'analyse de Balle perdue : espace approprié, conscientisé, sémiotisé, espace du vivre ensemble ou du projet, espace construit par le bas, par le haut, par le chercheur... la performance met à l'épreuve les différentes définitions du territoire, montrant son caractère flou, dynamique et fluctuant. La notion d'objet d'art-lieu, théorisée par Anne

39 Je ne suis en effet pas sûre que cette prise de conscience d'un passé, d'un présent et d'un futur commun soit aussi évidente pour un spectateur non averti des enjeux historiques, sociologiques ou urbanistiques de la zone d'implantation du MéPIC. 
Volvey à propos des pratiques spatiales de l'art contemporain, rend ainsi bien compte de ce qui se joue dans cette visite guidée: la création d'une œuvre-territoire aux résonances épistémologiques et la mise au jour du géographique comme pratique de création, de la spatialité comme matière artistique et de l'art comme moyen de produire des savoirs intéressant la géographie.

\section{Bibliographie}

Aucame, «La cité plateau. Une cité-jardin issu du paternalisme industriel », Qu'en savons-nous?, $\mathrm{n}^{\circ} 14$, juillet 2009.

Aucame, «La place du tourisme normand en France», Qu'en savons-nous?, $\mathrm{n}^{\circ} 34$, mai 2011.

Aucame, «Le port de Caen-Ouistreham», Qu'en savons-nous?, n 8, octobre 2008.

Caen, Conseil municipal de Caen, Caen, La CULTURE en capitales. Vivre et sémerveiller ensemble, communication sur le projet culturel de la Ville de Caen présentée au conseil municipal du 14 septembre 2009, [en ligne] http://www.caen.fr/culture/projetCulturel//, site consuté en septembre 2014.

Ariès, Philippe et Georges Duby (dir.), Histoire de la vie privée, t. 4: De la Révolution à la Grande Guerre, volume dirigé par Michelle Perrot, Paris, Seuil, 1987.

Austin, John Langshaw, Quand dire c'est faire, Paris, Seuil, 1970 [éd. anglaise, 1962].

Blanc, Nathalie, Vers une esthétique environnementale, Versailles, Éditions Quae, 2008.

Brunet, Roger, Robert Ferras et Hervé Théry (dir.), Les mots de la géographie, dictionnaire Critique, $2^{\mathrm{e}}$ éd. revue, Montpellier/Paris, GIP RECLUS/ La documentation française, 1992.

Charpy, Manuel, «Le théâtre des objets, Espaces privés, culture matérielle et identité bourgeoise. Paris 1830-1914», thèse d'histoire contemporaine, Tours, Université François Rabelais, 2010.

Cosset, Fabienne, Ma cabane en Normandie. Chalets, petites maisons et mobile homes du bord de mer, Caen, CRéCET, 2002. 
Dector, Michel et Michel Dupuy, Visite guidée du 19 juin 2013 Caen Ouistreham, Balle perdue, texte dactylographié, 2013, 8 p.

Dector et Dupuy, site internet de Dector et Dupuy, [en ligne] http://www. dector-dupuy.com/, site consulté en septembre 2014.

Elissalde, Bernard, «Une géographie des territoires», Linformation géographique, vol. 66, $n^{\circ}$ 3, 2002, p. 193-205.

"Estuaire 2009 Le paysage, l'art et le fleuve», 303 arts recherches créations, La revue culturelle des Pays de la Loire, $n^{\circ}$ 106, 2009.

«Estuaire 2012 Le paysage, l'art et le fleuve», 303 arts recherches créations, La revue culturelle des Pays de la Loire, $\mathrm{n}^{\circ}$ 122, 2012.

Eudes, Émeline, "The Romantic Geographic Society, ou comment et pourquoi marcher en Finlande en 2012. Retour sur l'œuvre d'itinérance de Jussi Kivi ", dans Laurent Buffet (dir.), Itinérances. L'art en déplacement, Grenoble, De l'incidence éditeur, 2012, p. 123-142.

Fleury, Adèle, «Comment la ville de Caen peut-elle pérenniser son tourisme patrimonial tout en diversifiant son offre touristique afin de créer une réelle destination touristique?", mémoire de master 2 en tourisme, Paris, Université de Paris 1, 2012.

Heinich, Nathalie et Roberta Shapiro (dir.), De l'artification. Enquêtes sur le passage à l'art, Paris, EHESS, 2012.

Houssay-Holzschuch, Myriam et Anne Volvey, «La rue comme palette. La Pietà sud-africaine, Soweto-Warwick, mai 2002, Ernest PignonErnest", Travaux de l'Institut de Géographie de Reims, n 129-130, 2008, p. 137-161.

Keerle, Régis et Raymonde Séchet, «Petite histoire de nos délicatesses avec le territoire", ESO, Travaux et documents de l'Unité Mixte de Recherche 6590, Espaces et sociétés, $\mathrm{n}^{\circ}$ 26, septembre 2007, p. 7-15.

"La presqu'île de Caen", Ohm, un petit journal de l'art contemporain (école régionale des Beaux-arts Caen-la-Mer), n 23, mars 2005.

Le Petit, Karine, Enquête ethnographique sur les métiers portuaires à Caen et Cherbourg, [en ligne] http://www.metiers-portuaires.fr/, consulté le 20 septembre 2014, 2014.

Le Thomas, Claire, «Art de l'entre-deux: le MéPIC au service de la reconversion portuaire de Caen", Urbanités, "Repenser la ville portuaire", $\mathrm{n}^{\circ} 4$, novembre 2014, [en ligne] http://www.revue-urbanites. $\mathrm{fr} /$, site consulté en septembre 2014, à paraître.

MéPIC, site internet du Musée éclaté de la presquîle de Caen, [en ligne] http://www.mepic.fr/, site consulté en septembre 2014, 2014. 
Société publique locale d'Aménagement (SPLA) Caen-Presqu'île (2014), site internet de la SPLA Caen-Presqu'̂̀le, [en ligne] http://www.caenpresquile.com/, site consulté en septembre 2014.

Vasset, Philippe, Un livre blanc. Récit avec cartes, Paris, Fayard, 2007.

Veschambre, Vincent, Traces et mémoires urbaines: enjeux sociaux de la patrimonialisation et de la démolition, Rennes, Presses universitaires de Rennes, 2008.

Volvey, Anne, «LAND ARTS. Les fabriques spatiales de l'art contemporain ", Travaux de l'Institut de Géographie de Reims, n 129-130, 2008, p. 3-25. 